\title{
Generalized Lie-algebraic structures related to integrable dispersionless dynamical systems and their application
}

\author{
Oksana E. Hentosh ${ }^{\mathrm{a}}$, Yarema A. Prykarpatsky ${ }^{\mathrm{b}}$, Denis Blackmore ${ }^{\mathrm{c}}$ and Anatolij Prykarpatski $^{\mathrm{d}{ }^{*}}$ \\ ${ }^{\mathrm{a}}$ The Institute for Applied Problems of Mechanics and Mathematics at the NAS, Lviv, 79060 Ukraine \\ ${ }^{\mathrm{b}}$ The Department of Applied Mathematics at the University of Agriculture in Krakow, 30059, Poland \\ ${ }^{\mathrm{c}}$ The Department of Mathematical Sciences at NJIT, University Heights, Newark, NJ 07102 USA \\ ${ }^{\mathrm{d}}$ The Department of Physics, Mathematics and Computer Science at the Krakow University of Technology, Krakow, 30-055, Poland \\ ${ }^{*}$ Corresponding author E-mail: pryk.anat@cybergal.com \\ Dedicated to our colleague, teacher, friend and brilliant mathematician Anatolij M. Samoilenko on his $80^{\text {th }}$ Birthday Jubilee
}

\begin{abstract}
Article Info
Keywords: Lax-Sato equations, Heavenly equations, Lax-Sato integrability, Hamiltonian system, Torus diffeomorphisms, Loop Lie algebra, Lie-algebraic scheme, Casimir invariants, R-structure, Lie-Poisson structure, Lagrange-d'Alembert principle

2010 AMS: $17 B 68,17 B 80,35 Q 53$, $35 G 25,35 N 10,37 K 35,58 J 70,58 J 72$, $34 \mathrm{~A} 34,37 \mathrm{~K} 05,37 \mathrm{~K} 10$

Received: 21 June 2018

Accepted: 20 September 2018

Available online: 30 September 2018
\end{abstract}

\begin{abstract}
Our review is devoted to Lie-algebraic structures and integrability properties of an interesting class of nonlinear dynamical systems called the dispersionless heavenly equations, which were initiated by Plebański and later analyzed in a series of articles. The AKS-algebraic and related $\mathscr{R}$-structure schemes are used to study the orbits of the corresponding co-adjoint actions, which are intimately related to the classical Lie-Poisson structures on them. It is demonstrated that their compatibility condition coincides with the corresponding heavenly equations under consideration. Moreover, all these equations originate in this way and can be represented as a Lax compatibility condition for specially constructed loop vector fields on the torus. The infinite hierarchy of conservations laws related to the heavenly equations is described, and its analytical structure connected with the Casimir invariants, is mentioned. In addition, typical examples of such equations, demonstrating in detail their integrability via the scheme devised herein, are presented. The relationship of a fascinating Lagrange-d'Alembert type mechanical interpretation of the devised integrability scheme with the Lax-Sato equations is also discussed. We pay a special attention to a generalization of the devised Lie-algebraic scheme to a case of loop Lie superalgebras of superconformal diffeomorphisms of the $1 \mid N$-dimensional supertorus. This scheme is applied to constructing the Lax-Sato integrable supersymmetric analogs of the Liouville and Mikhalev-Pavlov heavenly equation for every $N \in \mathbb{N} \backslash\{4 ; 5\}$.
\end{abstract}

\section{Introduction}

We shall discuss the Lax-Sato compatible systems, the related Lie-algebraic structures and complete integrability properties of an interesting class of nonlinear dynamical systems called the heavenly equations, introduced by Plebański [56] and analyzed in such articles as $[39,9,46,47,48,65,66,73,74]$. In our previous work, we employed the AKS-algebraic and related $\mathscr{R}$-structure schemes [6, 5, 7, 75, 62, 61], applied to the holomorphic Birkhoff type factorized loop Lie algebra $\tilde{\mathscr{G}}:=\widetilde{\operatorname{dif} f}\left(\mathbb{T}_{\mathbb{C}}^{1+n}\right)$ of vector fields on torus $\mathbb{T}_{\mathbb{C}}^{1+n} \simeq \mathbb{T}_{\mathbb{C}}^{1} \times \mathbb{T}^{n}, n \in \mathbb{Z}_{+}$, and reanalyzed and studied in detail the corresponding coadjoint actions on $\tilde{\mathscr{G}}^{*}$, closely related to the classical Lie-Poisson structures. By constructing two commuting flows on the coadjoint space $\tilde{\mathscr{G}}^{*}$, generated by a chosen root element $\tilde{l} \in \tilde{\mathscr{G}}^{*}$ and some Casimir invariants, we shall demonstrate that their compatibility condition coincides with the corresponding heavenly equations under consideration.

As a by-product of the construction devised recently in $[31,60]$, we prove that all the heavenly equations have a similar origin and can be represented as a Lax compatibility condition for special loop vector fields on the torus $\mathbb{T}_{\mathbb{C}}^{1+n}$. We analyze the structure of the infinite hierarchy of conservations laws related to the heavenly equations, and demonstrate that their analytical structure connected with the Casimir invariants is generated by the Lie-Poisson structure on $\tilde{\mathscr{G}}^{*}$. Moreover, we generalize the Lie-algebraic scheme of [31, 60] subject to the loop Lie algebra $\tilde{\mathscr{G}}^{*}=\widetilde{\operatorname{diff}}\left(\mathbb{T}_{\mathbb{C}}^{1 \mid N}\right)$ of superconformal vector fields on $\mathbb{T}_{\mathbb{C}}^{1 \mid N}$, which is the Lie algebra of the Lie group of superconformal diffeomorphisms of 
the $1 \mid N$-dimensional supertorus $\mathbb{T}_{\mathbb{C}}^{1 \mid N} \simeq \mathbb{T}_{\mathbb{C}}^{1} \times \Lambda_{1}^{N}$, where $\Lambda:=\Lambda_{0} \oplus \Lambda_{1}$ is an infinite-dimensional Grassmann algebra over $\mathbb{C}, \Lambda_{0} \supset \mathbb{C}$. This is applied to constructing the Lax-Sato type integrable superanalogs of the Mikhalev-Pavlov heavenly super-equation for every $N \in \mathbb{N} \backslash\{4 ; 5\}$. Typical examples are presented for all cases of the heavenly equations and their integrability is verified using the scheme devised here. This scheme also makes it possible to construct a very natural derivation of the well-known Lax-Sato type representation [63, 64] for an infinite hierarchy of heavenly equations, related to the canonical Lie-Poisson structure on the adjoint space $\tilde{\mathscr{G}}^{*}$. As a result of suitably chosen superconformal mappings in the space of variables $\left(z ; \theta_{1}, \ldots, \theta_{N}\right) \in \mathbb{T}_{\mathbb{C}}^{1 \mid N}$ the superanalogs of Liouville type equations are obtained. We also mention also show that an aspect of our approach to describing integrability of the heavenly dynamical systems is closely related to their classical Lagrange-d'Alembert type mechanical interpretation.

There are only a few examples of multi-dimensional integrable systems for which detailed descriptions of their mathematical structure have been given and our work is a next step in extending this list via new mathematical techniques utilizing the internal structure of heavenly nonlinear dispersionless integrable dynamical systems. We wish to mention that we have been strongly influenced both by the research of Pavlov, Bogdanov, Dryuma, Konopelchenko and Manakov [11, 9, 10, 33], as well as that of Ferapontov and Moss [26], Błaszak, Szablikowski and Sergyeyev, Krasil'shchik [7, 67, 70, 71, 68, 69, 34] wherein new effective differential-geometric and analytical methods for studying integrable degenerate multi-dimensional dispersionless heavenly type hierarchies of equations, the mathematical importance of which is still far from being fully appreciated.

\section{Generalized Lie-algebraic structures and related dispersionless heavenly type quasi-Hamiltonian systems}

\subsection{A generalized Lie algebra of holomorphic toral vector fields}

Let $\tilde{G}_{ \pm}:=\widetilde{\operatorname{Diff}} \pm\left(\mathbb{T}_{\mathbb{C}}^{1+n}\right), n \in \mathbb{Z}_{+}$, be subgroups of the Birkhoff type [57] loop diffeomorphisms group $\widetilde{\operatorname{Diff}}\left(\mathbb{T}_{\mathbb{C}}^{1+n}\right):=\left\{\mathbb{C} \supset \mathbb{S}^{1} \rightarrow\right.$ Diff hol $\left._{\text {hol }}\left(\mathbb{S}^{1} \times \mathbb{T}^{n}\right)\right\}$, holomorphically extended in the interior $\mathbb{D}_{+}^{1} \subset \mathbb{C}$ and in the exterior $\mathbb{D}_{-}^{1} \subset \mathbb{C}$ regions of the unit disc $\mathbb{D}^{1}$ with the boundary $\partial \mathbb{D}^{1}=\mathbb{S}^{1} \subset \mathbb{C}$, where for any $g(\lambda) \in \tilde{G}_{ \pm}$, either for $\lambda \in \mathbb{D}_{-}^{1}, g(\infty)=1 \in \operatorname{Diff}\left(\mathbb{T}_{\mathbb{C}}^{1+n}\right)$ or for $\lambda \in \mathbb{D}_{+}^{1}, g(0)=1 \in \operatorname{Diff}\left(\mathbb{T}_{\mathbb{C}}^{1+n}\right)$. The corresponding Lie subalgebras $\tilde{\mathscr{G}}_{ \pm}:=\widetilde{\operatorname{dif} f} f_{ \pm}\left(\mathbb{T}_{\mathbb{C}}^{1+n}\right)$ of the loop subgroups $\tilde{G}_{ \pm}$are vector fields on $\mathbb{T}_{\mathbb{C}}^{1+n}$ holomorphic, respectively, on $\mathbb{D}_{ \pm}^{1} \subset \mathbb{C}$, where either for any $\tilde{a}(\lambda) \in \tilde{\mathscr{G}}_{-}, \tilde{a}(\infty)=0$, or for any $\tilde{a}(\lambda) \in \tilde{\mathscr{G}}_{+}, \tilde{a}(0)=0$. The loop Lie algebra $\tilde{\mathscr{G}}:=\widetilde{\operatorname{diff}}\left(\mathbb{T}_{\mathbb{C}}^{1+n}\right)$ allows the direct sum splitting

$$
\tilde{\mathscr{G}}=\tilde{\mathscr{G}}_{+} \oplus \tilde{\mathscr{G}}_{-},
$$

which can be naturally identified with a dense subspace of the dual space $\tilde{\mathscr{G}}^{*}$ via the Sobolev type metric

$$
\left.(\tilde{l}, \tilde{a})_{s ; q}:=\underset{\lambda \in \mathbb{C}}{\operatorname{res}} \lambda^{-s} l(\lambda ; \mathrm{x}), a(\lambda ; \mathrm{x})\right)_{H^{q}}
$$

for some fixed $s \in \mathbb{Z}$ and $q \in \mathbb{Z}_{+}$. Here, by definition, a loop vector field $\tilde{a} \in \Gamma\left(\tilde{T}\left(\mathbb{T}_{\mathbb{C}}^{1+n}\right)\right)$ and a loop differential 1-form $\tilde{l} \in \tilde{\Lambda}^{1}\left(\mathbb{T}_{\mathbb{C}}^{1+n}\right)$ are given as

$$
\begin{gathered}
\tilde{a}=\sum_{j=1}^{n} a^{(j)}(\lambda ; x) \frac{\partial}{\partial x_{j}}+a^{(0)}(\lambda ; x):=\left\langle a(\mathrm{x}), \frac{\partial}{\partial \mathrm{x}}\right\rangle, \\
\tilde{l}=\sum_{j=1}^{n} l_{j}(\lambda ; x) d x_{j}+l_{0}(\lambda ; x):=\langle l(\mathrm{x}), d \mathrm{x}\rangle
\end{gathered}
$$

for any $\mathrm{x}:=(\lambda ; x) \in \mathbb{T}_{\mathbb{C}}^{1+n}$, and

$$
(l, a)_{H^{q}}=\sum_{j=0}^{n} \sum_{|\alpha|=0}^{q} \int_{\mathbb{T}^{n}} d x \frac{\partial^{|\alpha|} l_{j}}{\partial \mathrm{x}^{\alpha}} \frac{\partial^{|\alpha|} a_{j}}{\partial \mathrm{x}^{\alpha}} .
$$

In particular, for $q=0=s$ one has

$$
(\tilde{l}, \tilde{a})_{0}:=(\tilde{l}, \tilde{a})_{0 ; 0}=\underset{\lambda \in \mathbb{C}_{\mathbb{T}^{n}}}{\operatorname{ess}} \int_{j=0} d x\left(\sum_{j=0}^{n} l_{j} a_{j}\right),
$$

which is the case mainly chosen in the sequel.

It is now important to mention that the holomorphic Lie algebra $\tilde{\mathscr{G}}=\operatorname{diff}\left(\mathbb{T}_{\mathbb{C}}^{1+n}\right)$ splitting (2.1) makes it possible to introduce on the Lie algebra $\tilde{\mathscr{G}}$ the canonical $\mathscr{R}$-structure:

$$
[\tilde{a}, \tilde{b}]_{\mathscr{R}}:=[\mathscr{R} \tilde{a}, \tilde{b}]+[\tilde{a}, \mathscr{R} \tilde{b}]
$$

for any $\tilde{a}, \tilde{b} \in \tilde{\mathscr{G}}$, where

$$
\mathscr{R}:=\left(P_{+}-P_{-}\right) / 2,
$$

and

$$
P_{ \pm} \tilde{\mathscr{G}}:=\tilde{\mathscr{G}}_{ \pm} \subset \tilde{\mathscr{G}}
$$


Then for arbitrary smooth mappings $f, g \in \mathrm{D}\left(\tilde{\mathscr{G}}^{*}\right)$ one has two Lie-Poisson brackets

$$
\{f, g\}:=(\tilde{l},[\nabla f(\tilde{l}), \nabla g(l)])
$$

and

$$
\{f, g\}_{\mathscr{R}}:=\left(\tilde{l},[\nabla f(\tilde{l}), \nabla g(\tilde{l})]_{\mathscr{R}}\right),
$$

where at a fixed seed element $\tilde{l} \in \tilde{\mathscr{G}}^{*}$ the gradient elements $\nabla f(\tilde{l})$ and $\nabla g(\tilde{l}) \in \tilde{\mathscr{G}}$ are calculated, in general, with respect to the metric (2.4). Now let us assume that a smooth function $h \in \mathrm{I}\left(\tilde{\mathscr{G}}^{*}\right)$ is a Casimir invariant, that is

$$
a d_{\nabla h(\tilde{l})}^{*} \tilde{l}=0
$$

for a chosen seed element $\tilde{l} \in \tilde{\mathscr{G}}^{*}$. As the adjoint mapping $a d_{\nabla h(\tilde{l})}^{*}: \tilde{\mathscr{G}}^{*} \rightarrow \tilde{\mathscr{G}}^{*}$ for any $h \in D\left(\tilde{\mathscr{G}}^{*}\right)$ with respect to the scalar product (2.5) can be rewritten in the reduced form as

$$
a d_{\nabla h(\tilde{l})}^{*} \tilde{l}=\left\langle\frac{\partial}{\partial \mathrm{x}}, \circ \nabla h(l)\right\rangle \tilde{l}+\left\langle\left\langle l, \frac{\partial}{\partial \mathrm{x}} \nabla f(l)\right\rangle, d \mathrm{x}\right\rangle,
$$

where, by definition, $\nabla h(\tilde{l}):=<\nabla h(l), \frac{\partial}{\partial \mathrm{x}}>\in \tilde{\mathscr{G}}$ and “o" denotes the composition of mappings. For a suitable Casimir function $h \in D\left(\tilde{\mathscr{G}}^{*}\right)$, the condition (2.11) is then equivalent to the equation

$$
\left\langle\frac{\partial}{\partial \mathrm{x}}, \circ \nabla h(l)\right\rangle l+\left\langle l,\left(\frac{\partial}{\partial \mathrm{x}} \nabla h(l)\right)\right\rangle=0
$$

which should be be solved analytically. When $\tilde{l} \in \tilde{\mathscr{G}}^{*}$ is chosen to be singular as $|\lambda| \rightarrow \infty$, one can consider [18] the general asymptotic expansion

$$
\nabla h^{(p)}(l) \sim \lambda^{p} \sum_{j \in \mathbb{Z}_{+}} \eta_{j}^{(p)}(l) \lambda^{-j}
$$

for some suitably chosen integers $p \in \mathbb{Z}_{+}$, and upon substituting (2.14) into the equation (2.13), one can solve it recurrently. Now let $h^{\left(p_{y}\right)}, h^{\left(p_{y}\right)} \in \mathrm{I}\left(\tilde{\mathscr{G}}^{*}\right)$ be a Casimir functions for which the Hamiltonian vector field generators

$$
\nabla h^{(y)}(\tilde{l}):=\left.\nabla h^{\left(p_{y}\right)}(\tilde{l})\right|_{+}, \quad \nabla h^{(t)}(\tilde{l}):=\left.\nabla h^{\left(p_{t}\right)}(\tilde{l})\right|_{+}
$$

are, respectively, defined for special integers $p_{y}, p_{t} \in \mathbb{Z}_{+}$. These invariants generate, owing to the Lie-Poisson bracket (2.10), (as before for the case $q=0=s$ ) the following commuting flows

$$
\begin{aligned}
\partial l / \partial t & =-\left\langle\frac{\partial}{\partial \mathrm{x}}, \circ \nabla h^{(t)}(l)\right\rangle l-\left\langle l,\left(\frac{\partial}{\partial \mathrm{x}} \nabla h^{(t)}(l)\right)\right\rangle, \\
\partial l / \partial y & =-\left\langle\frac{\partial}{\partial \mathrm{x}}, \circ \nabla h^{(y)}(l)\right\rangle l-\left\langle l,\left(\frac{\partial}{\partial \mathrm{x}} \nabla h^{(y)}(l)\right)\right\rangle,
\end{aligned}
$$

where $y, t \in \mathbb{R}$ are the corresponding evolution parameters. Since the invariants $h^{\left(p_{y}\right)}, h^{\left(p_{y}\right)} \in \mathrm{I}\left(\tilde{\mathscr{G}}^{*}\right)$ commute with respect to the Lie-Poisson bracket (2.10), the flows (2.16) also commute, implying that the corresponding Hamiltonian vector field generators

$$
\nabla h^{(t)}(\tilde{l}):=\left\langle\nabla h^{(t)}(l), \frac{\partial}{\partial \mathrm{x}}\right\rangle, \quad \nabla h^{(y)}(\tilde{l}):=\left\langle\nabla h^{(y)}(l), \frac{\partial}{\partial \mathrm{x}}\right\rangle
$$

satisfy the Lax-Sato compatibility condition

$$
\frac{\partial}{\partial y} \nabla h^{(t)}(\tilde{l})-\frac{\partial}{\partial t} \nabla h^{(y)}(\tilde{l})=\left[\nabla h^{(t)}(\tilde{l}), \nabla h^{(y)}(\tilde{l})\right]
$$

for all $y, t \in \mathbb{R}$. On the other hand, the condition (2.18) is equivalent to the compatibility condition of two linear equations

$$
\left(\frac{\partial}{\partial t}+\nabla h^{(t)}(\tilde{l})\right) \psi=0, \quad\left(\frac{\partial}{\partial y}+\nabla h^{(y)}(\tilde{l})\right) \psi=0
$$

for a function $\psi \in C^{2}\left(\mathbb{R}^{2} \times \mathbb{T}_{\mathbb{C}}^{1+n} ; \mathbb{C}\right)$ and all $y, t \in \mathbb{R}$.

The above can be formulated as the following key result:

Proposition 2.1. Let a seed vector field be $\tilde{l} \in \tilde{\mathscr{G}}^{*}$ and $h^{\left(p_{y}\right)}, h^{\left(p_{y}\right)} \in \mathrm{I}\left(\tilde{\mathscr{G}}^{*}\right)$ be Casimir functions subject to the metric $(\cdot, \cdot)_{0}$ on the holomorphic Lie algebra $\tilde{\mathscr{G}}$ and the natural coadjoint action on the loop co-algebra $\tilde{\mathscr{G}}^{*}$. Then

$$
\partial \tilde{l} / \partial y=-a d_{\nabla h^{(y)}(\tilde{l})}^{*} \tilde{l}, \quad \partial \tilde{l} / \partial t=-a d_{\nabla h^{(t)}(\tilde{l})}^{*} \tilde{l}
$$

are commuting Hamiltonian dynamical systems for all $y, t \in \mathbb{R}$. Moreover, the compatibility condition of these flows is equivalent to the Lax-Sato vector field representation

$$
\left(\partial / \partial t+\nabla h^{(t)}(\tilde{l})\right) \psi=0, \quad\left(\partial / \partial y+\nabla h^{(y)}(\tilde{l})\right) \psi=0,
$$

where $\psi \in C^{2}\left(\mathbb{R}^{2} \times \mathbb{T}_{\mathbb{C}}^{1+n} ; \mathbb{C}\right)$ and the vector fields $\nabla h^{(t)}(\tilde{l}), \nabla h^{(t)}(\tilde{l}) \in \tilde{\mathscr{G}}$ are given by the expressions (2.17) and (2.15). 
Remark 2.2. As mentioned above, the expansion (2.14) is effective if a chosen seed element $\tilde{l} \in \tilde{\mathscr{G}}^{*}$ is singular as $|\lambda| \rightarrow \infty$. In the case when it is singular as $|\lambda| \rightarrow 0$, the expression (2.14) should be replaced by the expansion

$$
\nabla h^{(p)}(l) \sim \lambda^{-p} \sum_{j \in \mathbb{Z}_{+}} \eta_{j}^{(p)}(l) \lambda^{j}
$$

for suitably chosen integers $\quad p \in \mathbb{Z}_{+}$, and the reduced Casimir function gradients then are given by the Hamiltonian vector field generators

$$
\nabla h^{(y)}(l):=\left.\nabla h^{\left(p_{y}\right)}(l)\right|_{-}, \quad \nabla h^{(t)}(l):=\left.\nabla h^{\left(p_{t}\right)}(l)\right|_{-}
$$

for suitably chosen positive integers $p_{y}, p_{t} \in \mathbb{Z}_{+}$. Moreover, the corresponding Hamiltonian systems are, respectively, written as

$$
\partial \tilde{l} / \partial t=-a d_{\nabla h^{(t)}(\tilde{l})}^{*} \tilde{l}, \quad \partial \tilde{l} / \partial y=-a d_{\nabla h^{(y)}(\tilde{l})}^{*} l,
$$

where we need to mention that, owing to the analytical structure of the seed element $\tilde{l} \in \tilde{\mathscr{G}}^{*}$, the corresponding functional evolution equations on its coefficients are only quasi-Hamiltonian and are suitable reductions of the true Hamiltonian flows (2.24).

Remark 2.3. It is also possible to describe the Bäcklund transformations between two special solution sets for the dispersionless heavenly equations resulting from the Lax-Sato compatibility condition (2.20). In fact, take a diffeomorphism $\xi \in \widetilde{\operatorname{Diff}}\left(\mathbb{T}_{\mathbb{C}}^{1+n}\right)$ such that a seed differential form $\tilde{l}(\lambda ; x) \in \tilde{\mathscr{G}}^{*} \simeq \Lambda^{1}\left(\mathbb{T}_{\mathbb{C}}^{1+n}\right)$ satisfies the invariance condition

$$
\tilde{l}(\xi(\mathrm{x} \mid \mu))=k \tilde{l}(\overline{\mathrm{x}})
$$

for some non-zero constant $k \in \mathbb{C} \backslash\{0\}$, any $\mathrm{x}=(\lambda ; x)$ and $\overline{\mathrm{x}}=(\mu ; x) \in \mathbb{T}_{\mathbb{C}}^{1+n}$ with arbitrarily chosen parameter $\mu \in \mathbb{T}_{\mathbb{C}}^{1}$. As the seed element $\tilde{l}(\xi(\mathrm{x} \mid \mu)) \in \Lambda^{1}\left(\mathbb{T}_{\mathbb{C}}^{1+n}\right)$ satisfies the compatible equations $(2.20)$, the loop diffeomorphism $\xi \in \widetilde{\text { Diff }}\left(\mathbb{T}_{\mathbb{C}}^{1+n}\right)$, found analytically from the invariance condition (2.25), satisfies the compatible system of vector field equations

$$
\frac{\partial}{\partial t} \xi=\nabla h^{(t)}(l), \frac{\partial}{\partial y} \xi=\nabla h^{(y)}(l)
$$

giving rise to the Bäcklund type relationships for the coefficients of the seed differential form $\tilde{l} \in \tilde{\mathscr{G}}^{*} \simeq \Lambda^{1}\left(\mathbb{T}_{\mathbb{C}}^{1+n}\right)$.

The following examples demonstrate the analytical applicability of the above Lie-algebraic scheme for constructing a wide class of nonlinear multi-dimensional heavenly integrable Hamiltonian systems on function spaces.

\subsection{Example: Einstein-Weyl metric equation}

Define $\tilde{\mathscr{G}}:=\widetilde{\operatorname{diff}}\left(\mathbb{T}_{\mathbb{C}}^{2}\right)$, where for any $\tilde{a}(\lambda) \in \tilde{\mathscr{G}}_{-}, \lambda \in \mathbb{C}$, the value $\tilde{a}(\infty)=0$, and take a seed element $\tilde{l} \in \tilde{\mathscr{G}}^{*}$ in the following form

$$
\tilde{l}=\left(u_{x} \lambda-2 u_{x} v_{x}-u_{y}\right) d x+\left(\lambda^{2}-v_{x} \lambda+v_{y}+v_{x}^{2}\right) d \lambda,
$$

where $(u, v) \in C^{\infty}\left(\mathbb{R}^{2} \times \mathbb{T}^{1}\right)$ and which generates with respect to the metric (2.5) (as before for $q=0=s$ ) the gradients of the Casimir invariants $h^{(1)}, h^{(2)} \in \mathrm{I}\left(\tilde{\mathscr{G}}^{*}\right)$ in the form

$$
\begin{aligned}
& \nabla h^{(2)}(l) \quad \lambda^{2}(0,1)^{\top}+\left(-u_{x}, v_{x}\right)^{\top} \lambda+\left(u_{y}, u-v_{y}\right)^{\top}+O\left(\lambda^{-1}\right), \\
& \nabla h^{(1)}(l) \quad \sim \lambda(0,1)^{\top}+\left(-u_{x}, v_{x}\right)^{\top}+\left(u_{y},-v_{y}\right)^{\top} \lambda^{-1}+O\left(\lambda^{-2}\right)
\end{aligned}
$$

as $|\lambda| \rightarrow \infty$ at $p_{t}=2, p_{y}=1$. For the gradients of the Casimir functions $h^{(1)}, h^{(2)} \in \mathrm{I}\left(\mathscr{G}^{*}\right)$ determined by (2.15), one can easily obtain the corresponding Hamiltonian vector field generators

$$
\begin{gathered}
\nabla h^{(t)}(\tilde{l}):=\left\langle\nabla h^{(2)}(l)_{+}, \frac{\partial}{\partial \mathrm{x}}\right\rangle=\left(\lambda^{2}+\lambda v_{x}+u-v_{y}\right) \frac{\partial}{\partial x}+\left(-\lambda u_{x}+u_{y}\right) \frac{\partial}{\partial \lambda}, \\
\nabla h^{(y)}(\tilde{l})=\left\langle\nabla h^{(1)}(l)_{+}, \frac{\partial}{\partial \mathrm{x}}\right\rangle=\left(\lambda+v_{x}\right) \frac{\partial}{\partial x}-u_{x} \frac{\partial}{\partial \lambda},
\end{gathered}
$$

satisfying the compatibility condition (2.18), which is equivalent to the system

$$
\left\{\begin{array}{l}
u_{x t}+u_{y y}+\left(u u_{x}\right)_{x}+v_{x} u_{x y}-v_{y} u_{x x}=0 \\
v_{x t}+v_{y y}+u v_{x x}+v_{x} v_{x y}-v_{y} v_{x x}=0
\end{array}\right.
$$

describing general integrable Einstein-Weyl metric equations [23].

As is well known [39], the invariant reduction of (2.28) at $v=0$ gives rise to the famous dispersionless Kadomtsev-Petviashvili equation

$$
\left(u_{t}+u u_{x}\right)_{x}+u_{y y}=0,
$$

for which the reduced vector field representation (2.19) follows from (2.27) and is given by the vector fields

$$
\begin{gathered}
\nabla h^{(t)}(\tilde{l})=\left(\lambda^{2}+u\right) \frac{\partial}{\partial x}+\left(-\lambda u_{x}+u_{y}\right) \frac{\partial}{\partial \lambda}, \\
\nabla h^{(y)}(\tilde{l})=\lambda \frac{\partial}{\partial x}-u_{x} \frac{\partial}{\partial \lambda},
\end{gathered}
$$


satisfying the compatibility condition (2.18), equivalent to the equation (2.29). In particular, (2.19) and (2.30) imply the vector field compatibility relationships

$$
\begin{gathered}
\frac{\partial \psi}{\partial t}+\left(\lambda^{2}+u\right) \frac{\partial \psi}{\partial x}+\left(-\lambda u_{x}+u_{y}\right) \frac{\partial \psi}{\partial \lambda}=0 \\
\frac{\partial \psi}{\partial y}+\lambda \frac{\partial \psi}{\partial x}-u_{x} \frac{\partial \psi}{\partial \lambda}=0
\end{gathered}
$$

satisfied for $\psi \in C^{2}\left(\mathbb{R}^{2} \times \mathbb{T}_{\mathbb{C}}^{2} ; \mathbb{C}\right)$ and any $y, t \in \mathbb{R},(\lambda ; x) \in \mathbb{T}_{\mathbb{C}} \times \mathbb{T}^{1}$.

\subsection{Example: The modified Einstein-Weyl metric equation}

This equation system is

$$
\begin{gathered}
u_{x t}=u_{y y}+u_{x} u_{y}+u_{x}^{2} w_{x}+u u_{x y}+u_{x y} w_{x}+u_{x x} a, \\
w_{x t}=u w_{x y}+u_{y} w_{x}+w_{x} w_{x y}+a w_{x x}-a_{y},
\end{gathered}
$$

where $(u, w) \in C^{\infty}\left(\mathbb{R}^{2} \times \mathbb{T}^{1}\right), a_{x}:=u_{x} w_{x}-w_{x y}$, and was recently derived in [68]. In this case we also take $\tilde{\mathscr{G}}:=\widetilde{\operatorname{diff}}$ ( $\left.\mathbb{T}_{\mathbb{C}}^{2}\right)$, where for any $\tilde{a}(\lambda) \in \tilde{\mathscr{G}}_{-}, \lambda \in \mathbb{C}$, the value $\tilde{a}(\infty)=0$, yet for a seed element $\tilde{l} \in \tilde{\mathscr{G}}$ we choose the form

$$
\begin{gathered}
\tilde{l}=\left[\lambda^{2} u_{x}+\left(2 u_{x} w_{x}+u_{y}+3 u u_{x}\right) \lambda+2 u_{x} \partial_{x}^{-1} u_{x} w_{x}+2 u_{x} \partial_{x}^{-1} u_{y}+\right. \\
\left.+3 u_{x} w_{x}^{2}+2 u_{y} w_{x}+6 u u_{x} w_{x}+2 u u_{y}+3 u^{2} u_{x}-2 a u_{x}\right] d x+ \\
+\left[\lambda^{2}+\left(w_{x}+3 u\right) \lambda+2 \partial_{x}^{-1} u_{x} w_{x}+2 \partial_{x}^{-1} u_{y}+w_{x}^{2}+3 u w_{x}+3 u^{2}-a\right] d \lambda
\end{gathered}
$$

which with respect to the metric (2.5) (as before for $q=0=s$ ) generates two Casimir invariants $h^{(1)}, h^{(2)} \in \mathrm{I}\left(\tilde{\mathscr{G}}^{*}\right)$, whose gradients, as follows from (2.15), equal

$$
\begin{gathered}
\nabla h^{(2)}(l) \sim \lambda^{2}\left[\left(u_{x},-1\right)^{\top}+\left(u u_{x}+u_{y},-u+w_{x}\right)^{\top} \lambda^{-1}+\right. \\
\left.+\left(0, u w_{x}-a\right)^{\top} \lambda^{-2}\right]+O\left(\lambda^{-1}\right), \\
\nabla h^{(1)}(l) \quad \sim \lambda\left[\left(u_{x},-1\right)^{\top}+\left(0, w_{x}\right)^{\top} \lambda^{-1}\right]+O\left(\lambda^{-1}\right),
\end{gathered}
$$

as $|\lambda| \rightarrow \infty$ at $p_{y}=1, p_{t}=2$. The suitable positive projections

$$
\begin{gathered}
\nabla h^{(y)}(l):=\left.\nabla h^{(1)}(l)\right|_{+}=\left(u_{x} \lambda,-\lambda+w_{x}\right)^{\top}, \\
\nabla h^{(y)}(l) \quad:=\left.\nabla h^{(2)}(l)\right|_{+}=\left(u_{x} \lambda^{2}+\left(u u_{x}+u_{y}\right) \lambda,-\lambda^{2}+\left(w_{x}-u\right) \lambda+u w_{x}-a\right)^{\top} .
\end{gathered}
$$

of the gradients (2.34) generate the Hamiltonian flows (2.20), giving rise to the compatible Lax-Sato vector field system

$$
\begin{gathered}
\frac{\partial \psi}{\partial y}+\left(-\lambda+w_{x}\right) \frac{\partial \psi}{\partial x}+u_{x} \lambda \frac{\partial \psi}{\partial \lambda}=0 \\
\frac{\partial \psi}{\partial t}+\left[-\lambda^{2}+\left(w_{x}-u\right) \lambda+u w_{x}-a\right) \frac{\partial \psi}{\partial x}+\left(u_{x} \lambda^{2}+\left(u u_{x}+u_{y}\right) \lambda\right] \frac{\partial \psi}{\partial \lambda}=0,
\end{gathered}
$$

satisfied for $\psi \in C^{2}\left(\mathbb{R}^{2} \times \mathbb{T}_{\mathbb{C}}^{2} ; \mathbb{C}\right)$, any $y, t \in \mathbb{R}$ and all $(\lambda ; x) \in \mathbb{T}_{\mathbb{C}}^{2}$

\subsection{Example: The Dunajski heavenly equations}

This equation, suggested in [20], generalizes the corresponding anti-self-dual vacuum Einstein equation, which is related to the Plebański metric and the celebrated Plebański [56] second heavenly equation. To study the integrability of the Dunajski equations

$$
\begin{gathered}
u_{x_{1} t}+u_{y x_{2}}+u_{x_{1} x_{1}} u_{x_{2} x_{2}}-u_{x_{1} x_{2}}^{2}-v=0, \\
v_{x_{1} t}+v_{x_{2} y}+u_{x_{1} x_{1}} v_{x_{2} x_{2}}-2 u_{x_{1} x_{2}} v_{x_{1} x_{2}}=0,
\end{gathered}
$$

where $(u, v) \in C^{\infty}\left(\mathbb{R}^{2} \times \mathbb{T}^{2} ; \mathbb{R}^{2}\right),\left(y, t ; x_{1}, x_{2}\right) \in \mathbb{R}^{2} \times \mathbb{T}^{2}$, we define $\tilde{\mathscr{G}}:=\widetilde{\operatorname{dif} f}\left(\mathbb{T}_{\mathbb{C}}^{3}\right)$, where for any $\tilde{a}(\lambda) \in \tilde{\mathscr{G}}$ - the value $\tilde{a}(\infty)=0$, and take the following as a seed element $\tilde{l} \in \tilde{\mathscr{G}}^{*}$ :

$$
\tilde{l}=\left(\lambda+v_{x_{1}}-u_{x_{1} x_{1}}+u_{x_{1} x_{2}}\right) d x_{1}+\left(\lambda+v_{x_{2}}+u_{x_{2} x_{2}}-u_{x_{1} x_{2}}\right) d x_{2}+\lambda d \lambda .
$$


With respect to the metric (2.5) (as before for $q=0=s)$, the gradients of two functionally independent Casimir invariants $h^{\left(p_{y}\right)}, h^{\left(p_{t}\right)} \in \mathrm{I}\left(\tilde{\mathscr{G}}^{*}\right)$ can be obtained as $|\lambda| \rightarrow \infty$ in the asymptotic forms

$$
\begin{array}{ll}
\nabla h^{\left(p_{y}\right)}(l) & \sim \lambda(0,1,0)^{\top}+\left(-v_{x_{1}},-u_{x_{1} x_{2}}, u_{x_{1} x_{1}}\right)^{\top}+O\left(\lambda^{-1}\right) \\
\nabla h^{\left(p_{t}\right)}(l) & \sim \lambda(0,0,-1)^{\top}+\left(v_{x_{2}}, u_{x_{2} x_{2}},-u_{x_{1} x_{2}}\right)^{\top}+O\left(\lambda^{-1}\right)
\end{array}
$$

at $p_{t}=1=p_{y}$. Upon calculating the Hamiltonian vector field generators

$$
\begin{gathered}
\nabla h^{(y)}(l):=\left.\nabla h^{\left(p_{y}\right)}(l)\right|_{+}=\left(-v_{x_{1}}, \lambda-u_{x_{1} x_{2}}, u_{x_{1} x_{1}}\right)^{\top}, \\
\nabla h^{(t)}(l):=\left.\nabla h^{\left(p_{t}\right)}(l)\right|_{+}=\left(v_{x_{2}}, u_{x_{2} x_{2}},-\lambda-u_{x_{1} x_{2}}\right)^{\top},
\end{gathered}
$$

following from the Casimir functions gradients (2.39), one easily obtains the vector fields

$$
\begin{array}{r}
\nabla h^{(t)}(\tilde{l}):=<\nabla h^{(t)}(l), \frac{\partial}{\partial \mathrm{x}}>=u_{x_{2} x_{2}} \frac{\partial}{\partial x_{1}}-\left(\lambda+u_{x_{1} x_{2}}\right) \frac{\partial}{\partial x_{2}}+v_{x_{2}} \frac{\partial}{\partial \lambda}, \\
\nabla h^{(y)}(\tilde{l}) \quad:=<\nabla h^{(y)}(l), \frac{\partial}{\partial \mathrm{x}}>=\left(\lambda-u_{x_{1} x_{2}}\right) \frac{\partial}{\partial x_{1}}+u_{x_{1} x_{1}} \frac{\partial}{\partial x_{2}}-v_{x_{1}} \frac{\partial}{\partial \lambda},
\end{array}
$$

satisfying the Lax-Sato compatibility condition (2.18)

$$
\begin{aligned}
& \frac{\partial \psi}{\partial t}+u_{x_{2} x_{2}} \frac{\partial \psi}{\partial x_{1}}-\left(\lambda+u_{\left.x_{1} x_{2}\right)} \frac{\partial \psi}{\partial x_{2}}+v_{x_{2}} \frac{\partial \psi}{\partial \lambda}=0\right. \\
& \frac{\partial \psi}{\partial y}+\left(\lambda-u_{x_{1} x_{2}}\right) \frac{\partial \psi}{\partial x_{1}}+u_{x_{1} x_{1}} \frac{\partial \psi}{\partial x_{2}}-v_{x_{1}} \frac{\partial \psi}{\partial \lambda}=0
\end{aligned}
$$

equivalent to the the Dunajski [20] equation (2.37) and satisfied for $\psi \in C^{2}\left(\mathbb{R}^{2} \times \mathbb{T}_{\mathbb{C}}^{3} ; \mathbb{C}\right)$, any $\left(y, t ; x_{1}, x_{2}\right) \in \mathbb{R}^{2} \times \mathbb{T}^{2}$ and all $\lambda \in \mathbb{T}_{\mathbb{C}}^{1}$. As mentioned in [9], the Dunajski equations (2.37) generalize both the dispersionless Kadomtsev-Petviashvili and Plebański second heavenly equations, and is also a Lax-Sato integrable quasi-Hamiltonian system.

\subsection{Example: The Kupershmidt hydrodynamic heavenly type system}

This mutually compatible hydrodynamic system $[4,36,37,69,48]$ is given as

$$
\begin{gathered}
3 v_{y}-6 u v_{x}+6 u_{x} v+6 u u_{y}-6 u^{2} u_{x}-2 u_{t}=0 \\
-12 v_{x}+6 u_{y}-12 u u_{x}=0 \\
6 u v_{x x}-3 v_{x y}-6 u_{x x} v-6 u_{x} u_{y}+6 u^{2} u_{x x}-6 u u_{x y}+12 u u_{x}^{2}+2 u_{x t}=0 \\
6 v_{x x}+6 u u_{x x}-3 u_{x y}+6 u_{x}^{2}=0
\end{gathered}
$$

for smooth functions $(u, v) \in C^{\infty}\left(\mathbb{R}^{2} \times \mathbb{T}^{1} ; \mathbb{R}^{2}\right)$ with respect to "hidden" evolution parameters $t, y \in \mathbb{R}$ and the spatial variable $x \in \mathbb{T}^{1}$. Its Lax-Sato integrability stems from a seed element $\tilde{l} \in \tilde{\mathscr{G}}^{*}$, where $\tilde{\mathscr{G}}$ denotes the (holomorphic in $\lambda \in \mathbb{S}_{ \pm}^{1}$ ) Lie algebra $\tilde{\mathscr{G}}:=\widetilde{\operatorname{diff}}\left(\mathbb{T}_{\mathbb{C}}^{2}\right)$ of the loop diffeomorphism group $\widetilde{\operatorname{Diff}}\left(\mathbb{T}_{\mathbb{C}}^{2}\right)$, such that for any $\tilde{a}(\lambda) \in \tilde{\mathscr{G}}_{-}, \lambda \in \mathbb{C}, \tilde{a}(\infty)=0$ and

$$
\tilde{l}=\left[\lambda\left(v_{x}+2 u u_{x}\right)+\lambda^{2} u_{x}\right] d x+\left[\left(v+u^{2}\right)+2 \lambda u+\lambda^{2}\right] d \lambda
$$

for all $x \in \mathbb{T}^{1}$ and $\lambda \in \mathbb{T}_{\mathbb{C}}^{1}$. The corresponding gradients for the Casimir invariants $h^{(k)} \in \mathrm{I}\left(\tilde{\mathscr{G}}^{*}\right), k=\overline{1,2}$, are easily constructed from the determining conditions $a d_{\nabla h^{(k)}(\tilde{l})}^{*} \tilde{l}=0, k=\overline{1,2}$, as the following asymptotic expansions:

$$
\nabla h^{(k)}(l) \sim \lambda^{p_{k}} \sum_{j \in \mathbb{Z}_{+}} \eta_{j}^{(k)}(l) \lambda^{-j}
$$

giving rise at $p_{k}=k, k=\overline{1,2}$, to the expressions:

$$
\begin{gathered}
\nabla h^{(1)}(l) \quad \sim\left(2(\lambda+u),-2 \lambda u_{x}\right)^{\top}+O\left(\lambda^{-1}\right), \\
\nabla h^{(2)}(l) \quad \sim\left(3\left(\lambda^{2}+2 \lambda u++u^{2}+v\right),-3 \lambda\left(\lambda u_{x}+2 u u_{x}+v_{x}\right)\right)^{\top}+O\left(\lambda^{-1}\right),
\end{gathered}
$$

as $|\lambda| \rightarrow \infty$. Now taking into account the following Hamiltonian flows on $\tilde{\mathscr{G}}^{*}$

$$
d \tilde{l} / d y=-a d_{\nabla h^{(y)}(\tilde{l})}^{*} \tilde{l}, \quad d \tilde{l} / d y=-a d_{\nabla h^{(t)}(\tilde{l})}^{*} \tilde{l}
$$

with respect to the evolution parameters $y, t \in \mathbb{R}$, where, by definition,

$$
\nabla h^{(y)}(\tilde{l}):=\left.\nabla h^{(1)}(\tilde{l})\right|_{+}=2(\lambda+u) \partial / \partial x-2 \lambda u_{x} \partial / \partial \lambda,
$$

$$
\nabla h^{(t)}(\tilde{l}):=\left.\nabla h^{(2)}(\tilde{l})\right|_{+}=3\left(\lambda^{2}+2 \lambda u+u^{2}+v\right) \partial / \partial x-3 \lambda\left(\lambda u_{x}+2 u u_{x}+v_{x}\right) \partial / \partial \lambda
$$


are holomorphic vector fields on $\mathbb{T}_{\mathbb{C}}^{2}$, we can easily derive the corresponding compatible Kupershmidt hydrodynamic systems (2.43). It is also easy to check that the compatibility condition for a set of the vector fields (2.47) gives rise to the equivalent Lax-Sato vector field representation

$$
\begin{gathered}
\frac{\partial \psi}{\partial t}-3\left(\lambda^{2}+2 \lambda u+u^{2}+v\right) \frac{\partial \psi}{\partial x}+3 \lambda\left(\lambda u_{x}+2 u u_{x}+v_{x}\right) \frac{\partial \psi}{\partial \lambda}=0 \\
\frac{\partial \psi}{\partial y}-2(\lambda+u) \frac{\partial \psi}{\partial x}+2 \lambda u_{x} \frac{\partial \psi}{\partial \lambda}=0
\end{gathered}
$$

satisfied for $\psi \in C^{2}\left(\mathbb{R}^{2} \times \mathbb{T}_{\mathbb{C}}^{2} ; \mathbb{C}\right)$ for all $(y, t ; x, \lambda) \in \mathbb{R}^{2} \times \mathbb{T}_{\mathbb{C}}^{2}$. The result obtained above can be formulated as the follows:

Proposition 2.4. The Kupershmidt hydrodynamic heavenly system (2.43) is representable as commuting Hamiltonian flows (2.47) on orbits of the coadjoint action of the holomorphic loop Lie algebra $\tilde{\mathscr{G}}=\tilde{\operatorname{diff}}\left(\mathbb{T}_{\mathbb{C}}^{2}\right)$ and are equivalent to the Lax-Sato vector field compatibility condition (2.49).

\subsection{Examples:New spatially $3 D$-integrable heavenly systems}

\subsubsection{The first Sergyeyev spatially $3 D$-integrable heavenly system}

A new spatially $3 D$-integrable heavenly system, recently constructed in [71], using techniques from contact geometry [12, 38], is given as the flow

$$
\begin{aligned}
u_{t}-v_{y}-v u_{z}-r u_{x}+u v_{z}+w v_{x} & =0, \\
2 u_{z}-r_{z}+w_{x}+2 w w_{z} & =0, \\
2 r_{x}-3 u_{x}-2 w_{y}-v_{z}+2 w u_{z}-2 w w_{x}+2 u w_{z} & =0, \\
w_{t}-r_{y}+2 v_{x}-4 w u_{x}+w r_{x}-r w_{x}-v w_{z}+u r_{z} & =0
\end{aligned}
$$

with respect to two evolution parameters $t, y \in \mathbb{R}$ for four smooth functions $(u, v, w, r) \in C^{\infty}\left(\mathbb{R}^{2} \times \mathbb{T}^{2} ; \mathbb{R}^{4}\right)$. Let us set $\tilde{\mathscr{G}}:=\widetilde{\operatorname{dif} f}\left(\mathbb{T}_{\mathbb{C}}^{3}\right)$ and take the corresponding seed element $\tilde{l} \in \tilde{\mathscr{G}}^{*}$ as

$$
\begin{gathered}
\tilde{l}=\left[3 \partial _ { z } ^ { - 1 } \left(3 a_{x x x}+6 w_{z} a_{x x}+14 w_{x z} w_{x}+\left(12 w w_{x}+6 r_{x}\right) w_{z}+8 w w_{x x}+\right.\right. \\
\left.+\left(6 w^{2}+6 r\right) w_{x z}+16 w_{x}^{2}+6 r_{z} w_{x}+2 v_{x z}+r_{x x}+6 w r_{x z}\right)+ \\
\left.+6 \lambda a_{x x}+\left(36 \lambda a+12 \lambda^{2}\right) a_{x}+6 \lambda r_{x}\right] d x+ \\
+\left[27 a_{x x}+70 w_{z} a_{x}+\left(30 a^{2}+36 \lambda a+30 r+12 \lambda^{2}\right) w_{z}+\right. \\
\left.+(64 w+6 \lambda) w_{x}+10 v_{z}+(30 w+6 \lambda) r_{z}+9 r_{x}\right] d z+ \\
+\left[42 a_{x}+54 w^{2}+48 \lambda w+18 r+12 \lambda^{2}\right] d \lambda
\end{gathered}
$$

where $w_{x}=\left(r-w^{2}-2 u\right)_{z}$ and $a \in C^{\infty}\left(\mathbb{R}^{2} \times \mathbb{T}^{2} ; \mathbb{R}\right)$ is such that $w=a_{z}, r-w^{2}-2 u=a_{x}$ for all $x, z \in \mathbb{T}^{2}$. The seed element (2.51) naturally generates two independent Casimir functionals $h^{(1)}, h^{(2)} \in \mathrm{I}\left(\tilde{\mathscr{G}}^{*}\right)$, whose gradients allow as $|\lambda| \rightarrow \infty$ expansions (2.14) in the form

$$
\begin{gathered}
\nabla h^{(1)}(l) \sim\left(w_{z} \lambda^{2}+\left(u_{z}-w_{x}\right) \lambda-u_{x}, 2 \lambda+w, u-\lambda^{2}\right)^{\top}+O\left(\lambda^{-1}\right) \\
\nabla h^{(2)}(l) \sim\left(2 w_{z} \lambda^{3}+\left(-2 w_{x}+r_{z}\right) \lambda^{2}-r_{x}+v_{z}\right) \lambda-v_{x} \\
\left.3 \lambda^{2}+4 w \lambda+r,-2 \lambda^{3}-2 w \lambda^{2}+v\right)^{\top}+O\left(\lambda^{-1}\right)
\end{gathered}
$$

Now, by defining

$$
\begin{gathered}
\nabla h^{(y)}(l):=\left.\nabla h^{(1)}(l)\right|_{+}=\left(w_{z} \lambda^{2}+\left(u_{z}-w_{x}\right) \lambda-u_{x}, 2 \lambda+w, u-\lambda^{2}\right)^{\top}, \\
\nabla h^{(t)}(l):=\left.\nabla h^{(2)}(l)\right|_{+}=\left(2 w_{z} \lambda^{3}+\left(-2 w_{x}+r_{z}\right) \lambda^{2}-r_{x}+v_{z}\right) \lambda-v_{x}, \\
\left.3 \lambda^{2}+4 w \lambda+r,-2 \lambda^{3}-2 w \lambda^{2}+v\right)^{\top}
\end{gathered}
$$

subject to the canonical splitting $\tilde{\mathscr{G}}=\tilde{\mathscr{G}}_{+} \oplus \tilde{\mathscr{G}}_{-}$, one obtains for the heavenly equation (2.50) the following vector field representation

$$
\begin{gathered}
\frac{\partial \psi}{\partial t}+\left(3 \lambda^{2}+4 w \lambda+r\right) \frac{\partial \psi}{\partial x}+\left(-2 \lambda^{3}-2 w \lambda^{2}+v\right) \frac{\partial \psi}{\partial z}+ \\
+\left[2 w_{z} \lambda^{3}+\left(-2 w_{x}+r_{z}\right) \lambda^{2}+\left(-r_{x}+v_{z}\right) \lambda-v_{x}\right] \frac{\partial \psi}{\partial \lambda}=0, \\
\frac{\partial \psi}{\partial y}+[2 \lambda+w] \frac{\partial \psi}{\partial x}+\left(3 \lambda^{2}+4 w \lambda+r\right) \frac{\partial \psi}{\partial z}+\left(u-\lambda^{2}\right) \frac{\partial \psi}{\partial \lambda}=0,
\end{gathered}
$$

satisfied for $\psi \in C^{2}\left(\mathbb{R}^{2} \times \mathbb{T}_{\mathbb{C}}^{3} ; \mathbb{C}\right)$, any $(t, y ; x, z) \in \mathbb{R}^{2} \times \mathbb{T}^{2}$ and all $\lambda \in \mathbb{T}_{\mathbb{C}}$ 


\subsubsection{The second Sergyeyev spatially $3 D$-integrable heavenly system}

The second spatially 3-D integrable heavenly system, presented in [71], is given by two separate flows

$$
\begin{array}{cc}
u_{t} & =2 r v_{x}-2 v w_{z}+v r_{x}+w u_{x}, \\
v_{t} & =v w_{x}+w v_{x} \\
w_{y} & =2 v r_{z}-2 r u_{x}+u w_{z}+r v_{z} \\
r_{y} & =u r_{z}+r u_{z}
\end{array}
$$

with respect to two independent evolution parameters $t, y \in \mathbb{R}$ for four smooth functions $(u, v, w, r) \in C^{\infty}\left(\mathbb{R}^{2} \times \mathbb{T}^{2} ; \mathbb{R}^{4}\right)$. Inasmuch the system (2.54) is not completely specified as evolution flows and is obviously invariant with respect to the involution mapping $\operatorname{Symm}\{x \rightleftarrows z, t \rightleftarrows y ; u \rightleftarrows w, r \rightleftarrows v\}$, we need to take this into account to imbed this system into our Lie-algebraic integrability scheme.

We start, as before, from the basic vector fields Lie algebra $\widetilde{\operatorname{dif} f}\left(\mathbb{T}_{\mathbb{C}}^{3}\right)$ on the torus $\mathbb{T}_{\mathbb{C}}^{3}$ with coefficients from the differential-algebra $\mathbb{R}\{u, v, w, r \mid(x, z ; \lambda)\}$, considering its splitting

$$
\tilde{\mathscr{G}}:=\tilde{\mathscr{G}}_{+} \oplus \tilde{\mathscr{G}}
$$

with $\tilde{\mathscr{G}}_{ \pm}:=\left.\widetilde{\operatorname{dif} f}\left(\mathbb{T}_{\mathbb{C}}^{3}\right)\right|_{ \pm}$, naturally determining on $\tilde{\mathscr{G}}$ the canonical $\mathscr{R}$-structure $\mathscr{R}=\left(P_{+}-P_{-}\right) / 2$ with projections $P_{ \pm} \tilde{\mathscr{G}}:=\tilde{\mathscr{G}} \pm \subset(\tilde{\mathscr{G}}$. Taking into account the splitting (2.55), one can construct the adjoint with respect to the bilinear form $(\cdot, \cdot)_{0}$ space $\tilde{\mathscr{G}}^{*}=\tilde{\mathscr{G}}_{+}^{*} \oplus \tilde{\mathscr{G}}_{-}^{*}$, where $\tilde{\mathscr{G}}_{ \pm}^{*}=\left.\widetilde{\operatorname{diff}}^{*}\left(\mathbb{T}_{\mathbb{C}}^{3}\right)\right|_{ \pm}$. Now if we choose a generating seed element $\tilde{l} \in \tilde{\mathscr{G}}_{+}^{*} \oplus \tilde{\mathscr{G}}_{-}^{*}$ to be suitably anti-symmetric subject to the system (2.54), that is

$$
\operatorname{Symm}_{\lambda} \tilde{l}=-\tilde{l},
$$

where, by definition, the extended mapping $\operatorname{Symm}_{\lambda}: \tilde{\mathscr{G}}^{*} \rightarrow \tilde{\mathscr{G}}^{*}$ acts via the extended involution $\operatorname{Symm}_{\lambda}\{x \rightleftarrows z, t \rightleftarrows y ; u \rightleftarrows w, r \rightleftarrows v ; \lambda \rightleftarrows 1 / \lambda$ \} , then one can write down, as an example, the following expression

$$
\tilde{l}=l^{*}-\operatorname{Symm}_{\lambda} l^{*}
$$

with some element $l^{*} \in \tilde{\mathscr{G}}_{+}^{*}$ that we take in the form

$$
\begin{aligned}
l^{*}:= & r^{4} d \lambda++\left[r ^ { 2 } \frac { \partial } { \partial z } ^ { - 1 } \left(3 a r^{6} w_{z}-r w_{x x}+w r_{x x}+\right.\right. \\
& \left.\left.+r^{-1} w r_{x}^{2}+2 r^{-2} a_{x}\right)+a \lambda+r^{3} r_{x} \lambda^{2}\right] d x+ \\
+ & {\left[a-r^{3} w_{x}+2 r^{2} w r_{x}+r^{3}\left(w_{z}+r_{x}\right) \lambda+r^{3} r_{z} \lambda^{2}\right] d z }
\end{aligned}
$$

Here $a:=r^{3} \frac{\partial}{\partial z}^{-1}\left(3 r^{-1} r_{x} w_{z}+w_{x z}+r_{x x}+5 r^{-1} r_{x}^{2}\right)$, with coefficients from the suitably extended differential-algebra $\mathbb{R}\{u, v, w, r \mid(x, z ; \lambda)\}$ as it satisfies the Casimir determining equation (2.5) subject to the vector field

$$
\nabla h\left(l^{*}\right) \sim \nabla h^{(t)}\left(l^{*}\right)_{+}+\sum_{j \in \mathbb{Z}_{+}} \nabla h_{j}\left(l^{*}\right) \lambda^{-(j+1)},
$$

where

$$
\nabla h^{(t)}\left(l^{*}\right)=\left[r_{z} \lambda^{3}+\left(w_{z}-r_{x}\right) \lambda^{2}-w_{x} \lambda\right] \partial / \partial \lambda+(2 \lambda r+w) \partial / \partial x-r \lambda^{2} \partial / \partial z
$$

Based on the projected gradient element (2.60) one can construct on $\tilde{\mathscr{G}}^{*}$ the Hamiltonian flow

$$
\partial l^{*} / \partial t=-a d_{\nabla h^{(t)}\left(l^{*}\right)}^{*}
$$

with respect to the temporal evolution parameter $t \in \mathbb{R}$. Recalling now the symmetry property (2.56), one can apply the mapping Symm $\lambda$ to (2.60) and obtain, as a result, the evolution flow

$$
\partial l^{*} / \partial y=-a d_{\nabla h^{(y)}\left(l^{*}\right)}^{*} l^{*}
$$

with respect to the evolution parameter $y \in \mathbb{R}$, which is compatible with the flow (2.61), where we define

$$
\nabla h^{(y}\left(l^{*}\right):=\operatorname{Symm}_{\lambda} \nabla h^{(t)}\left(l^{*}\right)=\left(\lambda u_{z}-u_{x}+v_{z}-v_{x} \lambda^{-1}\right) \partial / \partial \lambda-v \lambda^{-2} \partial / \partial x+\left(u+2 v \lambda^{-1}\right) \partial / \partial z .
$$

Having applied now the mapping $\operatorname{Symm}_{\lambda}$ to both sides of the relationship (2.62), we obtain the flow

$$
\frac{\partial}{\partial t}\left(\operatorname{Symm}_{\lambda} l^{*}\right)=-a d_{\nabla h^{(t)}\left(l^{*}\right)}^{*}\left(\operatorname{Symm}_{\lambda} l^{*}\right) .
$$

Combining this with (2.61) gives rise to the flow

$$
\partial \tilde{l} / \partial t=-a d_{\nabla h^{(t)}\left(l^{*}\right)}^{*} \tilde{l}
$$


which is a priori compatible with its symmetry $\operatorname{Symm}_{\lambda}$ image:

$$
\partial \tilde{l} / \partial y=-a d_{\nabla h^{(y)}\left(l^{*}\right)}^{\tilde{l}} \text {. }
$$

As the evolution parameters are mutually independent, from flows (2.61) and (2.62) one obtains their natural compatibility condition, which is equivalent to the following vector field expression:

$$
\frac{\partial}{\partial t} \nabla h^{(y)}\left(l^{*}\right)-\frac{\partial}{\partial y} \nabla h^{(t)}\left(l^{*}\right)+\left[\nabla h^{(t)}\left(l^{*}\right), \nabla h^{(y)}\left(l^{*}\right)\right]=0,
$$

giving rise to the initial heavenly system of equations of equations (2.54). As a simple consequence of (2.67), we obtain for the heavenly system (2.54) its Lax-Sato compatible vector fields representation

$$
\begin{aligned}
& \partial \psi / \partial t+\left[r_{z} \lambda^{3}+\left(w_{z}-r_{x}\right) \lambda^{2}-w_{x} \lambda\right] \partial \psi / \partial \lambda+(2 \lambda r+w) \partial \psi / \partial x-r \lambda^{2} \partial \psi / \partial z=0 \\
& \partial \psi / \partial y+\left(\lambda u_{z}-u_{x}+v_{z}-v_{x} \lambda^{-1}\right) \partial \psi / \partial \lambda-v \lambda^{-2} \partial \psi / \partial x+\left(u+2 v \lambda^{-1}\right) \partial \psi / \partial z=0
\end{aligned}
$$

satisfied for an invariant $\psi \in C^{2}\left(\mathbb{R}^{2} \times \mathbb{T}_{\mathbb{C}}^{3} ; \mathbb{C}\right)$, any $(t, y ; x, z) \in \mathbb{R}^{2} \times \mathbb{T}^{2}$ and all $\lambda \in \mathbb{T}_{\mathbb{C}}$.

Remark 2.5. It must be mentioned that the vector field $\nabla h^{(y}\left(l^{*}\right) \in \tilde{\mathscr{G}}$ as devised above, possesses no proto-Casimir functionals $h_{j}^{(y)} \in$ $\mathrm{D}\left(\tilde{\mathscr{G}}^{*}\right), j=\overline{1,2}$, whose gradients projections $\left.\nabla h_{j}^{(y)}\left(l^{*}\right)\right|_{ \pm} \in \tilde{\mathscr{G}}_{ \pm}, j=\overline{1,2}$, would be generated. That is $\nabla h^{(y}\left(l^{*}\right) \neq\left.\nabla h_{1}^{(y)}\left(l^{*}\right)\right|_{+}+\left.\nabla h_{2}^{(y)}\left(l^{*}\right)\right|_{-}$ for any smooth functionals $h_{j}^{(y)} \in \mathrm{D}\left(\tilde{\mathscr{G}}^{*}\right), j=\overline{1,2}$.

\subsection{Example: A generalized Liouville type equation}

In [10], devoted to studying Grassmannians, closed differential forms and related $N$-dimensional integrable systems, the authors presented a Lax-Sato type representation for the well-known Liouville equation

$$
\frac{\partial^{2} \varphi}{\partial y \partial t}=\exp \varphi
$$

written in the so called "laboratory" coordinates $y, t \in \mathbb{R}^{2}$ for a function $\varphi \in C^{2}\left(\mathbb{R}^{2} ; \mathbb{R}\right)$ and having different geometric interpretations. Their related result, obtained via some completely formal calculations, reads as follows: a system of the linear vector field equations

$$
\begin{aligned}
\partial \psi / \partial y+\left(\lambda^{2}+v \lambda+1\right) \partial \psi / \partial \lambda & =0, \\
\partial \psi / \partial t-u \partial \psi / \partial \lambda & =0
\end{aligned}
$$

for a function $\psi \in C^{2}\left(\mathbb{R}^{2} \times \mathbb{T}_{\mathbb{C}}^{1} ; \mathbb{C}\right)$ is compatible for all $y, t \in \mathbb{R}^{2}$, where $u, v \in C^{2}\left(\mathbb{R}^{2} ; \mathbb{R}\right)$ are functional coefficients and $\lambda \in \mathbb{T}_{\mathbb{C}}^{1}$ is a complex parameter. Under the simple reduction $u=1 / 2 \exp \varphi$ the compatibility condition for (2.70) coincides with the Liouville equation (2.69).

This section is devoted to unveiling the Lie-algebraic structure of a generalized Liouville type heavenly equation, whose Lax-Sato integrability was shown in [10] using geometric analysis of Grassmanians and related closed differential forms.

As our interest is in the Lie-algebraic nature of the Lax-Sato representation (2.70) for the Liouville equation (2.69), we define the torus diffeomorphism Lie group $\tilde{G}:=\widetilde{\operatorname{Diff}}\left(\mathbb{T}_{\mathbb{C}}^{1}\right)$, holomorphically extended in the interior $\mathbb{D}_{+}^{1} \subset \mathbb{C}$ and in the exterior $\mathbb{D}_{-}^{1} \subset \mathbb{C}$ regions of the unit disc $\mathbb{D}^{1} \subset \mathbb{C}^{1}$, such that for any $\left.g(z) \in \bar{G}\right|_{\mathbb{D}_{-}^{1}}, z \in \mathbb{D}_{-}^{1}, g(\infty)=1 \in \widetilde{\operatorname{Diff}}\left(\mathbb{T}_{\mathbb{C}}^{1}\right)$. Then we study specially chosen coadjoint orbits, which are related to the compatible system of linear vector field equations (2.70).

As a first step,one needs to consider the corresponding Lie algebra $\tilde{\mathscr{G}}:=\widetilde{\operatorname{diff}}\left(\mathbb{T}_{\mathbb{C}}^{1}\right)$ and its decomposition into the direct sum of subalgebras

$$
\tilde{\mathscr{G}}=\tilde{\mathscr{G}}_{+} \oplus \tilde{\mathscr{G}}_{-}
$$

of Laurent series with positive as $|z| \rightarrow 0$ and strongly negative as $|z| \rightarrow \infty$ degrees, respectively. Then, owing to classical Adler-Kostant-Symes theory, for any element $\tilde{l} \in \tilde{\mathscr{G}}^{*} \simeq \Lambda^{1}\left(\mathbb{T}_{\mathbb{C}}^{1}\right)$ the following formally constructed flows

$$
d \tilde{l} / d y=-a d_{\nabla h^{(y)}(\tilde{l})}^{*} \tilde{l}, \quad d \tilde{l} / d t=-a d_{\nabla h^{(t)}(\tilde{l})}^{*} \tilde{l}
$$

along the evolution parameters $y, t \in \mathbb{R}^{2}$ are always compatible, if $h^{\left(p_{y}\right)}$ and $h^{\left(p_{t}\right)} \in \mathrm{I}\left(\tilde{\mathscr{G}}^{*}\right)$ are arbitrarily chosen functionally independent Casimir functionals on the adjoint space $\tilde{\mathscr{G}}^{*}$, and $\nabla h^{(y)}(\tilde{l}):=\nabla h^{\left(p_{y}\right)}(\tilde{l})_{+}, \nabla h^{(t)}(\tilde{l}):=\nabla h^{\left(p_{t}\right)}(\tilde{l})_{+}$are their gradients, suitably projected on the subalgebra $\tilde{\mathscr{G}}_{+}$. Keeping in mind the above result, consider the Casimir functional $h^{\left(p_{y}\right)}$ on $\tilde{\mathscr{G}}^{*}$, whose gradient $\nabla h^{\left(p_{y}\right)}(\tilde{l}):=\nabla h^{\left(p_{y}\right)}(l) \partial / \partial z$ as $|z| \rightarrow \infty$ is taken, for simplicity, in the asymptotic form

$$
\nabla h^{\left(p_{y}\right)}(\tilde{l}) \simeq\left(v_{2} z^{2}+v_{1} z+v_{0}+v_{-1} z^{-1}+v_{-2} z^{-2}+\ldots\right) \partial / \partial z,
$$

where $p_{y}=2$. This gives rise to the gradient projection $\nabla h^{(y)}(\tilde{l})=\left(v_{2} z^{2}+v_{1} z+v_{0}\right) \partial / \partial z \in \tilde{\mathscr{G}}_{+}$, where $z \in \mathbb{T}_{\mathbb{C}}^{1},|z| \rightarrow \infty$, and $v_{j} \in$ $C^{2}\left(\mathbb{R}^{2} ; \mathbb{R}\right), j \in \mathbb{Z}, j \leq 2$, are some functional parameters. As the element $\tilde{l}=l(y, t ; z) d z \in \Lambda^{1}\left(\mathbb{T}_{\mathbb{C}}^{1}\right)$ satisfies, by definition, the differential equation

$$
\frac{d}{d z}\left[l(y, t ; z)\left(\nabla h^{\left(p_{y}\right)}(l)\right)^{2}\right]=0
$$


we obtain from (2.74) that the element

$$
l(y, t ; z)=\sigma(y, t)^{2}\left(\nabla h^{\left(p_{y}\right)}(\tilde{l})\right)^{-2},
$$

where $\sigma \in C^{2}\left(\mathbb{R}^{2} ; \mathbb{R}\right)$ is an arbitrary function. If for brevity we set $\sigma(y, t):=1$ and $v_{2}:=1$, the element (2.75) becomes

$$
l(y, t ; z)=z^{-4}\left[1-2 v_{1} z^{-1}+\left(3 v_{1}^{2}-2 v_{0}\right) z^{-2}\right]
$$

Observe now that the relationship (2.74) verifies the following lemma:

Lemma 2.6. The set $\mathrm{I}\left(\tilde{\mathscr{G}}^{*}\right)$ of the functionally independent Casimir invariants is one-dimensional.

As a consequence, it follows that for the element $\tilde{l}=l(y, t ; z) d z \in \Lambda^{1}\left(\mathbb{T}_{\mathbb{C}}^{1}\right)$ generated by the expression (2.75), there exists only the flow on $\tilde{\mathscr{G}}^{*}$ from (2.72) with respect to the evolution variable $y \in \mathbb{R}$ :

$$
d l / d y=\nabla h^{(y)}(l)^{-1} \frac{\partial}{\partial z}\left[l(y, t ; z) \nabla h^{(y)}(l)\right]^{2} .
$$

For the flow from (2.72) with respect to the evolution variable $t \in \mathbb{R}$ one can take the constant functional $h^{\left(p_{t}\right)}:=$ const $\in \mathrm{I}\left(\tilde{\mathscr{G}}^{*}\right), p_{t}=$ $0, \nabla h^{\left(p_{t}\right)}(l)=0$, and construct the trivial flow on $\tilde{\mathscr{G}}^{*}$ as

$$
d l / d t=-\nabla h^{(t)}(l) \frac{\partial l}{\partial z}-2 l \frac{\partial}{\partial z}\left(\nabla h^{(t)}(l)\right)=0,
$$

where, by definition, $\nabla h^{(t)}(l):=\nabla h^{\left(p_{t}\right)}(l)_{+} \in \tilde{\mathscr{G}}$.It is now important to observe that the compatibility condition of these two flows for all $y, t \in \mathbb{R}$ is equivalent to the following system of two a priori compatible linear vector field equations

$$
\frac{\partial \psi}{\partial y}+\nabla h^{(y)}(l) \frac{\partial \psi}{\partial z}=0, \quad \frac{\partial \psi}{\partial t}+\nabla h^{(t)}(l) \frac{\partial \psi}{\partial z}=0
$$

or

$$
\frac{\partial \psi}{\partial y}+\left(z^{2}+v_{1} z+v_{0}\right) \frac{\partial \psi}{\partial z}=0, \quad \frac{\partial \psi}{\partial t}+0 \frac{\partial \psi}{\partial z}=0
$$

for a smooth function $\psi \in C^{2}\left(\mathbb{R}^{2} \times \mathbb{T}_{\mathbb{C}}^{1} ; \mathbb{C}\right)$, meaning, in particular, that the complex parameter $z \in \mathbb{T}_{\mathbb{C}}^{1}$ is constant with respect to the evolution parameter $t \in \mathbb{R}$. The linear equations (2.80) are clearly equivalent to the $a$ priori compatible system of the vector fields

$$
d z / d y=\nabla h^{(y)}(l)=z^{2}+v_{1} z+v_{0}, \quad d z / d t=\nabla h^{(t)}(l)=0
$$

on the complex torus $\mathbb{T}_{\mathbb{C}}^{1}$, which can be rewritten subject to the following diffeomorphism $\mathbb{T}_{\mathbb{C}}^{1} \ni z \mapsto z-\alpha(t, y):=\lambda \in \mathbb{T}_{\mathbb{C}}^{1}$, generated by an arbitrary smooth function $\alpha \in C^{3}\left(\mathbb{R}^{2} ; \mathbb{R}\right)$ :

$$
d \lambda / d y=\lambda^{2}+\lambda\left(2 \alpha+v_{1}\right)+\left(\alpha^{2}+\alpha v_{1}+v_{0}-\partial \alpha / \partial y\right), \quad d \lambda / d t=-\partial \alpha / \partial t .
$$

The latter system is evidently also compatible for all $y, t \in \mathbb{R}$ and can be expressed as

$$
d \lambda / d y=\lambda^{2}+\lambda v+w, d \lambda / d t=-u,
$$

where

$$
2 \alpha+v_{1}:=v, \alpha^{2}+\alpha v_{1}+v_{0}-\partial \alpha / \partial y:=w, \quad \partial \alpha / \partial t:=u .
$$

Moreover, the a priori compatible system (2.80) can be recast as

$$
\frac{\partial \psi}{\partial y}+\left(z^{2}+v z+w\right) \frac{\partial \psi}{\partial \lambda}=0, \quad \frac{\partial \psi}{\partial y}-u \frac{\partial \psi}{\partial \lambda}=0
$$

for the corresponding function $\psi \in C^{2}\left(\mathbb{R}^{2} \times \mathbb{T}_{\mathbb{C}}^{1} ; \mathbb{C}\right)$, giving rise to the following system of heavenly equations:

$$
v_{t}-2 u=0, \quad u_{y}-u v+w_{t}=0 .
$$

The latter can be parameterized by means of the substitution $u:=1 / 2 \exp \varphi$ as follows:

$$
\varphi_{y t}=\exp \varphi-\left[2 w_{t} \exp (-\varphi)\right]_{t} .
$$

Then the reductions $w:=$ const $=1$ or $w:=-\frac{1}{2} \exp \varphi$ give rise to the well-known Liouville equations

$$
\varphi_{y t}=\exp \varphi, \quad \varphi_{y t}-\varphi_{t t}=\exp \varphi,
$$

which are known to possess standard $[6,44,59]$ Lax type isospectral representations. The above analysis leads directly to the following result.

Proposition 2.7. The system (2.86) of heavenly nonlinear equations possesses Lax-Sato type compatible vector field representation (2.85), whose Lie-algebraic structure is governed by the classical Lie-algebraic Adler-Kostant-Symes theory.

Remark 2.8. In a manner like the above, one can describe in detail the Lie-algebraic structure for other generalized Liouville type heavenly equations, presented in [10] for a higher order in $\lambda \in \mathbb{T}_{\mathbb{C}}^{1}$ system of linear vector field equations (2.79). 


\section{The linearization covering method and its applications}

\subsection{Introductory notions and examples}

Some three years ago I. Krasilshchik [34] analyzed a so called Gibbon-Tsarev equation

$$
z_{y y}+z_{t} z_{t y}-z_{y} z_{t t}+1=0 .
$$

and its so called nonlinear first-order differential covering

$$
\frac{\partial w}{\partial t}-\frac{1}{z_{y}+z_{t} w-w^{2}}=0, \frac{\partial w}{\partial y}+\frac{z_{t}-w}{u_{y}+z_{t} w-w^{2}}=0,
$$

which for any solution $z: \mathbb{R}^{2} \rightarrow \mathbb{R}$ to equation (3.1) is compatible for all $(t, y) \in \mathbb{R}^{2}$. He showed this makes it possible to determine for any smooth solution $w: \mathbb{R}^{2} \rightarrow \mathbb{R}$ to the equation (3.2) a suitable smooth function $\psi: \mathbb{R} \times \mathbb{R}^{2} \rightarrow \mathbb{R}$, satisfying the corresponding Lax-Sato type linear representation:

$$
\frac{\partial \psi}{\partial t}+\frac{1}{z_{y}+z_{t} \lambda-\lambda^{2}} \frac{\partial \psi}{\partial \lambda}=0, \frac{\partial \psi}{\partial y}-\frac{z_{t}-\lambda}{z_{y}+z_{t} \lambda-\lambda^{2}} \frac{\partial \psi}{\partial \lambda}=0
$$

which for any solution to the equation (3.1) is also compatible for all $(t, y) \in \mathbb{R}^{2}$ and an arbitrary parameter $\lambda \in \mathbb{R}$.

Krasilshchik [34] also posed the interesting problem of providing a differential-geometric explanation of the linearization procedure for a given nonlinear differential-geometric relationship $\left.J^{1}\left(\mathbb{R}^{n} \times \mathbb{R}^{2} ; \mathbb{R}\right)\right|_{\mathscr{E}}$ in the jet-manifold $J^{1}\left(\mathbb{R}^{n} \times \mathbb{R}^{2} ; \mathbb{R}\right), n \in \mathbb{Z}_{+}$, realizing a compatible covering for the corresponding nonlinear differential equation $\mathscr{E}[x, \tau ; u]=0$, imbedded into some adjacent jet-manifold $J^{k}\left(\mathbb{R}^{n} \times \mathbb{R}^{2} ; \mathbb{R}^{m}\right)$ for some $k, m \in \mathbb{Z}_{+}$. His extended version of this procedure, presented in [34], was quite hard to decipher and offered no new example demonstrating its application. One of our goals is to explain some important points of this linearization procedure in the framework of the classical nonuniform vector field equations and present new and important applications. We consider the jet manifold $J^{k}\left(\mathbb{R}^{n} \times \mathbb{R}^{2} ; \mathbb{R}^{m}\right)$ for some fixed $k, m \in \mathbb{Z}_{+}$and a differential relationship [30] in a general form $\mathscr{E}[x, \tau ; u]=0$, satisfied for all $(x ; \tau) \in \mathbb{R}^{n} \times \mathbb{R}^{2}$ and suitable smooth mappings $u: \mathbb{R}^{n} \times \mathbb{R}^{2} \rightarrow \mathbb{R}^{m}$.

As a new example, we can take $n=1=m, k=2$ and choose a differential relationship $\mathscr{E}[x ; y, t ; u]=0$ in the form

$$
u_{t} u_{x y}-k_{1} u_{x} u_{t y}-k_{2} u_{y} u_{t x}=0
$$

the so called ABC-equation, first discussed in [76], where $u: \mathbb{R} \times \mathbb{R}^{2} \rightarrow \mathbb{R}$ and $k_{1}, k_{2} \in \mathbb{R}$ are arbitrary parameters, satisfying the conditions

$$
k_{1}+k_{2}-1=0 \vee k_{1}+k_{2}-1 \neq 0 .
$$

The first case $k_{1}+k_{2}-1=0$ was investigated in [22, 42, 76] and recently in [31], where its Lax-Sato type linearization was found along with many other its interesting properties. For the second case $k_{1}+k_{2}-1 \neq 0$ the following crucial result was stated in equivalent form by P.A. Burovskiy, E.V. Ferapontov, S.P. Tsarev in [16, 35] and recently by I. Krasilshchik, A. Sergyeyev and O. Morozov in [35].

Proposition 3.1. A dual to (3.4) covering system $\left.J^{1}\left(\mathbb{R} \times \mathbb{R}^{2} ; \mathbb{R}\right)\right|_{\mathscr{E}}$ of quasi-linear first order differential relationships

$$
\begin{aligned}
& \frac{\partial w}{\partial t}+\frac{u_{t} w}{u_{x} k_{1}\left(k_{1}+k_{2}-1\right)} \frac{\partial w}{\partial x}-\frac{w\left(w+k_{1}+k_{2}-1\right) u_{t x}}{u_{x} k_{1}}=0, \\
& \frac{\partial w}{\partial y}+\frac{u_{y} w}{u_{x} k_{1}\left(w+k_{1}+k_{2}-1\right)} \frac{\partial w}{\partial x}-\frac{w\left(k_{1}+k_{2}-1\right) u_{y x}}{u_{x} k_{1}}=0
\end{aligned}
$$

on the jet-manifold $J^{1}\left(\mathbb{R} \times \mathbb{R}^{2} ; \mathbb{R}\right)$ is compatible; that is, it holds for any its smooth solution $w: \mathbb{R} \times \mathbb{R}^{2} \rightarrow \mathbb{R}$ at all points $(x ; y, t) \in \mathbb{R} \times \mathbb{R}^{2}$ iff the function $u: \mathbb{R} \times \mathbb{R}^{2} \rightarrow \mathbb{R}$ satisfies the $A B C$-equation (3.4).

Moreover, this result was recently generalized in [35] to the following "linearizing" proposition.

Proposition 3.2. A system $\left.J_{\text {lin }}^{1}\left(\mathbb{R}^{2} \times \mathbb{R}^{2} ; \mathbb{R}\right)\right|_{\mathscr{E}}$ of linear first order differential relationships

$$
\begin{aligned}
& \frac{\partial \psi}{\partial t}+\frac{\lambda u_{x}^{\frac{k_{2}-1}{k_{1}}} u_{t}}{k_{1}\left(k_{1}+k_{2}-1\right)} \frac{\partial \psi}{\partial x}-\frac{\lambda^{2} u_{x}^{\frac{k_{2}-k_{1}-1}{k_{1}}}\left(u_{t} u_{x x}-k_{1} u_{x x} u_{x t}\right)}{k_{1}^{2}} \frac{\partial \psi}{\partial \lambda}=0 \\
& \frac{\partial \psi}{\partial y}+\frac{k_{2} \lambda u_{x}^{\frac{k_{2}-1}{k_{1}}}}{u_{y}} \frac{\partial \psi}{k_{1}\left(\lambda u_{x}^{\frac{k_{2}-k_{1}-1}{k_{1}}}+k_{1}+k_{2}-1\right)}-\frac{\lambda^{2} k_{2}\left(k_{1}+k_{2}-1\right) u_{x}^{\frac{k_{2}-k_{1}-1}{k_{1}}} u_{y} u_{x x}}{\partial x} \frac{\partial \psi}{\partial \lambda}=0
\end{aligned}
$$

on the covering jet-manifold $J^{1}\left(\mathbb{R}^{2} \times \mathbb{R}^{2} ; \mathbb{R}\right)$ is compatible; that is, it holds for any its smooth solution $\psi: \mathbb{R}^{2} \times \mathbb{R}^{2} \rightarrow \mathbb{R}$ at all points $(x, \lambda ; y, t) \in \mathbb{R}^{2} \times \mathbb{R}^{2}$ iff the function $u: \mathbb{R} \times \mathbb{R}^{2} \rightarrow \mathbb{R}$ satisfies the generalized ABC-equation (3.4).

A similar result, when $n=1, m, k=2$, was proved for the Manakov-Santini equations

$$
\begin{aligned}
u_{t x}+u_{y y}+\left(u u_{x}\right)_{x}+v_{x} u_{x y}-v_{y} u_{x x} & =0, \\
v_{x t}+v_{y y}+u v_{x x}+v_{x} u_{x y}-v_{y} v_{x x} & =0,
\end{aligned}
$$


whose Lax-Sato integrability was extensively studied in $[21,24,39,11,31]$. The system (3.8) as a jet-submanifold $\left.J^{1}\left(\mathbb{R} \times \mathbb{R}^{2} ; \mathbb{R}\right)\right|_{\mathscr{E}} \subset$ $J^{1}\left(\mathbb{R} \times \mathbb{R}^{2} ; \mathbb{R}\right)$ allows the following nonlinear first order differential representation

$$
\begin{gathered}
\frac{\partial w}{\partial t}+\left(w^{2}-w v_{x}+u-v_{y}\right) \frac{\partial w}{\partial x}+u_{x} w-u_{y}+v_{y y}+v_{x}\left(v_{y}-u\right)_{x}=0, \\
\frac{\partial w}{\partial y}+w \frac{\partial w}{\partial x}-v_{x x} w+\left(u-v_{y}\right)_{x}=0
\end{gathered}
$$

compatible on solutions to the nonlinear differential relationship $\mathscr{E}[x, \tau ; u]=0(3.8)$ on $J^{2}\left(\mathbb{R} \times \mathbb{R}^{2} ; \mathbb{R}^{2}\right)$. The existence of the compatible representation (3.9) makes it possible to verify the following proposition.

Proposition 3.3. A covering system $\left.J_{\text {lin }}^{1}\left(\mathbb{R}^{2} \times \mathbb{R}^{2} ; \mathbb{R}\right)\right|_{\mathscr{E}}$ of linear first order differential relationships

$$
\begin{gathered}
\frac{\partial \psi}{\partial t}+\left(\lambda^{2}+\lambda v_{x}+u-v_{y}\right) \frac{\partial \psi}{\partial x}+\left(u_{y}-\lambda u_{x}\right) \frac{\partial \psi}{\partial \lambda}=0, \\
\frac{\partial \psi}{\partial y}+\left(v_{x}+\lambda\right) \frac{\partial \psi}{\partial x}-u_{x} \frac{\partial \psi}{\partial \lambda}=0
\end{gathered}
$$

on the jet-manifold $J^{1}\left(\mathbb{R}^{2} \times \mathbb{R}^{2} ; \mathbb{R}\right)$ is compatible; that is, it holds for any its smooth solution $\psi: \mathbb{R}^{2} \times \mathbb{R}^{2} \rightarrow \mathbb{R}$ at all points $(x, \lambda ; y, t) \in$ $\mathbb{R}^{2} \times \mathbb{R}^{2}$ iff the function $u: \mathbb{R} \times \mathbb{R}^{2} \rightarrow \mathbb{R}$ satisfies the generalized Khokhlov-Zabolotska equation (3.8).

From the point of view of the propositions above, the main essence of our present analysis, similarly to that in [34], is to recover the intrinsic mathematical structure responsible for the existence of the "linearizing" covering jet-manifold mappings

$$
\left.\left.J_{\text {lin }}^{1}\left(\mathbb{R}^{n+1} \times \mathbb{R}^{2} ; \mathbb{R}\right)\right|_{\mathscr{E}} \rightleftharpoons J^{1}\left(\mathbb{R}^{n} \times \mathbb{R}^{2} ; \mathbb{R}\right)\right|_{\mathscr{E}}
$$

for any dimension $n \in \mathbb{Z}_{+}$, compatible with our differential relationship $\mathscr{E}[x, \tau ; u]=0$, as it was presented above in the form (3.6) and (3.7) for the relationships (3.4). Thus, for a given nonlinear differential relationship $\mathscr{E}[x, \tau ; u]=0$ on the jet-manifold $J^{k}\left(\mathbb{R}^{n} \times \mathbb{R}^{2} ; \mathbb{R}^{m}\right)$ for some $k \in \mathbb{Z}_{+}$one can formulate the following problem:

If there is a compatible system $\left.J^{1}\left(\mathbb{R}^{n} \times \mathbb{R}^{2} ; \mathbb{R}^{m}\right)\right|_{\mathscr{E}} \subset J^{1}\left(\mathbb{R}^{n} \times \mathbb{R}^{2} ; \mathbb{R}^{m}\right)$ of quasi-linear first order differential relationships, how can construct a linearizing first order differential system $\left.J_{\text {lin }}^{1}\left(\mathbb{R}^{(1+n)+2} ; \mathbb{R}\right)\right|_{\mathscr{E}} \subset J^{1}\left(\mathbb{R}^{(1+n)+2} ; \mathbb{R}\right)$ in a vector field equations form on the covering space $\mathbb{R}^{n+1} \times \mathbb{R}^{2}$, realizing the implications (3.11). The latter is interpreted as the corresponding Lax-Sato representation $[8,9,10,24,25,73,74]$ for the given differential relationship $\mathscr{E}[x, \tau ; u]=0$ on the jet-manifold $J^{k}\left(\mathbb{R}^{n} \times \mathbb{R}^{2} ; \mathbb{R}^{m}\right)$.

As a dual approach to this linearization covering scheme, we present also the so called contact geometry linearization, suggested recently in [71] and slightly generalizing the well-known [69] Hamiltonian linearization covering method. As an example, we have proved the following proposition.

Proposition 3.4. The following [16] nonlinear singular manifold Toda differential relationship

$$
u_{x y} s h^{2} u_{t}=u_{x} u_{y} u_{t t}
$$

on the jet manifold $J^{2}\left(\mathbb{R}^{2} \times \mathbb{R}^{2} ; \mathbb{R}\right)$ allows the Lax-Sato type linearization covering

$$
\begin{array}{r}
\frac{\partial \psi}{\partial t}+\frac{\left(e^{-2 u_{t}}-1\right)}{2 u_{x}} \frac{\partial \psi}{\partial x}-\left[\lambda\left(\frac{e^{-2 u_{t}}-1}{2 u_{x}}\right)_{x}+\lambda^{2}\left(\frac{e^{-2 u_{t}}-1}{2 u_{x}}\right)_{z}\right] \frac{\partial \psi}{\partial \lambda}=0 \\
\frac{\partial \psi}{\partial y}-\frac{u_{y} e^{-2 u_{t}}}{u_{x}} \frac{\partial \psi}{\partial x}+\left[\lambda\left(\frac{u_{y} e^{-2 u_{t}}}{u_{x}}\right)_{x}+\lambda^{2}\left(\frac{u_{y} e^{-2 u_{t}}}{u_{x}}\right)_{z}\right] \frac{\partial \psi}{\partial \lambda}=0
\end{array}
$$

for smooth invariant functions $\psi \in C^{2}\left(\mathbb{R}^{3} \times \mathbb{R}^{2} ; \mathbb{R}\right)$, all $(x, z, \lambda ; \tau) \in \mathbb{R}^{3} \times \mathbb{R}^{2}$ and any smooth solution $u: \mathbb{R}^{2} \times \mathbb{R}^{2} \rightarrow \mathbb{R}$ to the relationship (3.12).

\subsection{The linearization covering scheme}

A realization of the scheme (3.11) is based on the notion of invariants of suitably specified vector fields on the extended base space $\mathbb{R}^{n+1} \times \mathbb{R}^{2}$, whose definition suitable for our needs is as follows: a smooth mapping $\psi: \mathbb{R}^{n+1} \times \mathbb{R}^{2} \rightarrow \mathbb{R}$ is, subject to parameters $\tau \in \mathbb{R}^{2}$, an invariant of a set of vector fields

$$
X^{(k)}:=\frac{\partial}{\partial \tau_{k}}+\sum_{j=\overline{1, n}} a_{j}^{(k)}(x, \lambda ; \tau) \frac{\partial}{\partial x_{j}}+b^{(k)}(x, \lambda ; \tau) \frac{\partial}{\partial \lambda}
$$

on $\mathbb{R}^{n+1} \times \mathbb{R}^{2}$ with smooth coefficients $\left(a^{(k)}, b^{(k)}\right): \mathbb{R}^{n+1} \times \mathbb{R}^{2} \rightarrow \mathbb{E}^{n} \times \mathbb{R}, k=\overline{1,2}$, if

$$
X^{(k)} \psi=0
$$

holds for $k=\overline{1,2}$ and all $(x, \lambda ; \tau) \in \mathbb{R}^{n+1} \times \mathbb{R}^{2}$. The system of linear equations (3.15) is equivalently representable as a jet-submanifold $\left.J_{\text {lin }}^{1}\left(\mathbb{R}^{n+1} \times \mathbb{R}^{2} ; \mathbb{R}\right)\right|_{\mathscr{E}} \subset J^{1}\left(\mathbb{R}^{n+1} \times \mathbb{R}^{2} ; \mathbb{R}\right)$. It is also well known [17] that simultaneously the following vector field flows

$$
\frac{\partial x_{j}}{\partial \tau_{k}}=a_{j}^{(k)}(x, \lambda ; \tau), \frac{\partial \lambda}{\partial \tau_{k}}=b^{(k)}(x, \lambda ; \tau)
$$


are compatible for any $j=\overline{1, n}, k=\overline{1,2}$ and all $(x, \lambda ; \tau) \in \mathbb{R}^{n+1} \times \mathbb{R}^{2}$. Taking now into account that there is such an invariant function $\psi: \mathbb{R}^{n+1} \times \mathbb{R}^{2} \rightarrow \mathbb{R}$ representable as $\psi(x, \lambda ; \tau)=w(x ; \tau)-\lambda:=0$ for some smooth mapping $w: \mathbb{R}^{n} \times \mathbb{R}^{2} \rightarrow \mathbb{R}$, it provides upon its substitution into (3.15) the following a priori compatible reduced system of quasilinear first order equations

$$
\frac{\partial w}{\partial \tau_{k}}+\sum_{j=\overline{1, n}} a_{j}^{(k)}(x, w ; \tau) \frac{\partial w}{\partial x_{j}}-b^{(k)}(x, w ; \tau)=0
$$

for $k=\overline{1,2}$ on the jet-manifold $J^{0}\left(\mathbb{R}^{n} \times \mathbb{R}^{2} ; \mathbb{R}\right)$. Moreover, subject to the system (3.17) one sees [17, 29] that, modulo solutions to the equations (3.16), the expression $w(x ; \tau)=\psi(x, \lambda(\tau) ; \tau)+\lambda(\tau)$ for all $(x ; \tau) \in \mathbb{R}^{n} \times \mathbb{R}^{2}$, where $\psi: \mathbb{R}^{n+1} \times \mathbb{R}^{2} \rightarrow \mathbb{R}$ is a first integral of the vector field flows (3.16). Thus, the reduction scheme just described above provides the algorithm

$$
\left.\left.J_{\text {lin }}^{1}\left(\mathbb{R}^{n+1} \times \mathbb{R}^{2} ; \mathbb{R}\right)\right|_{\mathscr{E}} \quad \rightarrow \quad J^{1}\left(\mathbb{R}^{n} \times \mathbb{R}^{2} ; \mathbb{R}\right)\right|_{\mathscr{E}}
$$

from the implications (3.11) formulated above. The corresponding inverse implication

$$
\left.\left.J_{\text {lin }}^{1}\left(\mathbb{R}^{n+1} \times \mathbb{R}^{2} ; \mathbb{R}\right)\right|_{\mathscr{E}} \quad \leftarrow \quad J^{1}\left(\mathbb{R}^{n} \times \mathbb{R}^{2} ; \mathbb{R}\right)\right|_{\mathscr{E}}
$$

can be algorithmically described as follows.

Consider a compatible system $\left.J^{1}\left(\mathbb{R}^{n+2} ; \mathbb{R}\right)\right|_{\mathscr{E}} \subset J^{1}\left(\mathbb{R}^{n+2} ; \mathbb{R}\right)$ of the first order nonlinear differential relationships

$$
\frac{\partial w}{\partial \tau_{k}}+\sum_{j=1, n} a_{j}^{(k)}(x, w ; \tau) \frac{\partial w}{\partial x_{j}}-b^{(k)}(x, w ; \tau)=0
$$

with smooth coefficients $\left(a^{(k)}, b^{(k)}\right): \mathbb{R}^{n+1} \times \mathbb{R}^{2} \rightarrow \mathbb{E}^{n} \times \mathbb{R}, k=\overline{1,2}$. As the first step it is necessary to check whether the adjacent system of vector field flows

$$
\frac{\partial x_{j}}{\partial \tau_{k}}=a_{j}^{(k)}(x, w ; \tau)
$$

on $\mathbb{R}^{n+1}$ modulo the flows (3.20) for all $j=\overline{1, n}$ and $k=\overline{1,2}$ is also compatible. If the answer is yes, it just means [17] that any solution to (3.20) as a complex function $w: \mathbb{R}^{n} \times \mathbb{R}^{2} \rightarrow \mathbb{R}$ is representable as $w(x ; \tau)-\lambda=\alpha(\psi(x, \lambda ; \tau))$ for any $\lambda \in \mathbb{R}$ and some smooth mapping $\alpha: \mathbb{R} \rightarrow \mathbb{R}$, where the mapping $\psi: \mathbb{R}^{n+1} \times \mathbb{R}^{2} \rightarrow \mathbb{R}$ is a first integral of the vector field equations

$$
\frac{\partial \psi}{\partial \tau_{k}}+\sum_{j=\overline{1, n}} a_{j}^{(k)}(x, \lambda ; \tau) \frac{\partial \psi}{\partial x_{j}}+b^{(k)}(x, \lambda ; \tau) \frac{\partial \psi}{\partial \lambda}=0
$$

on the extended space $\mathbb{R}^{n+1} \times \mathbb{R}$ for all $(x, \lambda ; \tau) \in \mathbb{R}^{n+1} \times \mathbb{R}^{2}$. Moreover, the value $w(x(\tau) ; \tau)=\lambda \in \mathbb{R}$ for all $\tau \in \mathbb{R}^{2}$ is constant as follows from the condition $\alpha(\psi(x(\tau), w ; \tau))=0$ for the $\tau \in \mathbb{R}^{2}$. Thus, we have shown that the equations (3.22) realize the covering linear first order differential relationships $\left.J_{\text {lin }}^{1}\left(\mathbb{R}^{n+1} \times \mathbb{R}^{2} ; \mathbb{R}\right)\right|_{\mathscr{E}} \subset J^{1}\left(\mathbb{R}^{n+1} \times \mathbb{R}^{2} ; \mathbb{R}\right)$ for $k=\overline{1,2}$ and all $(x, \lambda ; \tau) \in \mathbb{R}^{n+1} \times \mathbb{R}^{2}$, linearizing the first order nonlinear equations (3.20) and interpreting it as the corresponding Lax-Sato representation.

On the other hand, if the adjacent system of vector field flows (3.21) is not compatible, it is necessary to recover a hidden isomorphic transformation

$$
J^{1}\left(\mathbb{R}^{n+1} \times \mathbb{R}^{2} ; \mathbb{R}\right) \ni(x, w ; \tau) \rightarrow(x, \tilde{w} ; \tau) \in J^{1}\left(\mathbb{R}^{n+1} \times \mathbb{R}^{2} ; \mathbb{R}\right),
$$

for which the resulting a priori compatible first order equations

$$
\frac{\partial \tilde{w}}{\partial \tau_{k}}+\sum_{j=\overline{1, n}} \tilde{a}_{j}^{(k)}(x, \tilde{w} ; \tau) \frac{\partial \tilde{w}}{\partial x_{j}}-\tilde{b}^{(k)}(x, \tilde{w} ; \tau)=0
$$

already possess a compatible adjacent system of the corresponding flows

$$
\frac{\partial x_{j}}{\partial \tau_{k}}=\tilde{a}_{j}^{(k)}(x, \tilde{w} ; \tau)
$$

on the space $\mathbb{R}^{n} \times \mathbb{R}$, for which any solution $\tilde{w}: \mathbb{R}^{n+2} \rightarrow \mathbb{R}$ generates a first integral $\tilde{\psi}: \mathbb{R}^{n+1} \times \mathbb{R}^{2} \rightarrow \mathbb{R}$ of an adjacent compatible system of the linear vector field equations

$$
\frac{\partial \tilde{\psi}}{\partial \tau_{k}}+\sum_{j=\overline{1, n}} \tilde{a}_{j}^{(k)}(x, \lambda ; \tau) \frac{\partial \tilde{\psi}}{\partial x_{j}}+\tilde{b}^{(k)}(x, \lambda ; \tau) \frac{\partial \tilde{\psi}}{\partial \lambda}=0
$$

on the space $\mathbb{R}^{n+1} \times \mathbb{R}^{2} \rightarrow \mathbb{R}$ for $k=\overline{1,2}$. Here $\tilde{\psi}(x, \lambda ; \tau):=\tilde{\alpha}(\tilde{w}(x ; \tau)-\lambda)$ for all $(x, \lambda ; \tau) \in \mathbb{R}^{n+1} \times \mathbb{R}$ and some smooth mapping $\tilde{\alpha}: \mathbb{R} \rightarrow$ $\mathbb{R}$. From this one easily obtains - as above - a linearized covering jet-submanifold $\left.J_{\text {lin }}^{1}\left(\mathbb{R}^{n+1} \times \mathbb{R}^{2} ; \mathbb{R}\right)\right|_{\mathscr{E}} \subset J^{1}\left(\mathbb{R}^{n+1} \times \mathbb{R}^{2} ; \mathbb{R}\right)$, as a compatible system of the vector field equations (3.26), generated by the nonlinear first order differential system $\left.J^{1}\left(\mathbb{R}^{n} \times \mathbb{R}^{2} ; \mathbb{R}\right)\right|_{\mathscr{E}} \subset J^{1}\left(\mathbb{R}^{n} \times \mathbb{R}^{2} ; \mathbb{R}\right)$ on the space $\mathbb{R}^{n} \times \mathbb{R}^{2}$. This determines the inverse implication (3.19) as applied to general compatible first order equations (3.22), providing for the nonlinear first order system $\left.J^{1}\left(\mathbb{R}^{n} \times \mathbb{R}^{2} ; \mathbb{R}\right)\right|_{\mathscr{E}} \subset J^{1}\left(\mathbb{R}^{n} \times \mathbb{R}^{2} ; \mathbb{R}\right)$ its corresponding Lax-Sato representation. 
Remark 3.5. The existence of the map (3.23) can be deduced from the following reasoning. Assume that the mapping (3.23) exists and is equivalent to

$$
w(x ; \tau):=\rho(x, \tilde{w}(x ; \tau) ; \tau)
$$

for some smooth function $\rho: \mathbb{R}^{n+1} \times \mathbb{R}^{2} \rightarrow \mathbb{R}$ and all $(x ; \tau) \in \mathbb{R}^{n} \times \mathbb{R}^{2}$, where the corresponding map $\tilde{w}: \mathbb{R}^{n} \times \mathbb{R}^{2} \rightarrow \mathbb{R}$ satisfies the following system of differential equations:

$$
\frac{\partial \tilde{w}}{\partial \tau_{k}}+\sum_{j=\overline{1, n}} a_{j}^{(k)}(x, \rho(x, \tilde{w} ; \tau) ; \tau) \frac{\partial \tilde{w}}{\partial x_{j}}=\tilde{b}^{(k)}(x, \tilde{w} ; \tau),
$$

compatible for all $\tau_{k} \in \mathbb{R}, k=\overline{1,2}$, and $x \in \mathbb{R}^{n}$. Here functions $\tilde{b}^{(k)}: \mathbb{R}^{n+1} \times \mathbb{R}^{2} \rightarrow \mathbb{R}, k=\overline{1,2}$, defined as

$$
\tilde{b}^{(k)}(x, \tilde{w} ; \tau):=\left.\left[b^{(k)}-\sum_{j=\overline{1, n}}\left(\frac{\partial \rho}{\partial \tau_{k}}+a_{j}^{(k)} \frac{\partial \rho}{\partial x_{j}}\right)\right]\left(\frac{\partial \rho}{\partial x_{j}}\right)^{-1}\right|_{w=\rho(x, \tilde{w} ; \tau)},
$$

should depend on the mapping (3.27) in such a way that the vector fields

$$
\frac{\partial x_{j}}{\partial \tau_{k}}=a_{j}^{(k)}(x, \rho(x, \tilde{w} ; \tau) ; \tau):=\tilde{a}_{j}^{(k)}(x, \tilde{w} ; \tau)
$$

are also compatible for all $j=\overline{1, n}$ and $k=\overline{1,2}$ modulo the flows (3.28). This means that the equation (3.28) can be equivalently represented as a compatible system of the following vector field equations

$$
\frac{\partial \tilde{\psi}}{\partial \tau_{k}}+\sum_{j=\overline{1, n}} \tilde{a}_{j}^{(k)}(x, \lambda ; \tau) \frac{\partial \tilde{\psi}}{\partial x_{j}}+\tilde{b}^{(k)}(x, \rho(x, \lambda ; \tau) ; \tau) \frac{\partial \tilde{\psi}}{\partial \lambda}=0
$$

on its first integral $\tilde{\psi}: \mathbb{R}^{n+1} \times \mathbb{R}^{2} \rightarrow \mathbb{R}$, where $\tilde{\psi}(x, \lambda ; \tau)=\alpha(\tilde{w}(x ; \tau)-\lambda)$ for an arbitrarily chosen smooth mapping $\alpha: \mathbb{R} \rightarrow \mathbb{R}$, any parameter $\lambda \in \mathbb{R}$ and all $(x ; \tau) \in \mathbb{R}^{n} \times \mathbb{R}^{2}$. The system (3.31) provides a suitable Lax-Sato type linearization of the compatible quasi-linear first order differential equations (3.17). Concerning the map (3.27) and its dependents on it functions $\tilde{b}^{(k)}: \mathbb{R}^{n+1} \times \mathbb{R}^{2} \rightarrow \mathbb{R}, k=\overline{1,2}$, one can easily observe that the compatibility condition for the vector fields (3.30) reduces to the a priori compatible equations

$$
\begin{gathered}
\frac{\partial a_{j}^{(k)}}{\partial \rho} \frac{\partial \rho}{\partial \tau_{s}}-\frac{\partial a_{j}^{(s)}}{\partial \rho} \frac{\partial \rho}{\partial \tau_{k}}+\left(\frac{\partial a_{j}^{(k)}}{\partial \rho} \tilde{b}^{(s)}-\frac{\partial a_{j}^{(s)}}{\partial \rho} \tilde{b}^{(k)}\right) \frac{\partial \rho}{\partial \tilde{w}}+ \\
+\sum_{m=\overline{1, n}}\left(\frac{\partial a_{j}^{(k)}}{\partial \rho} a_{m}^{(s)}-\frac{\partial a_{j}^{(s)}}{\partial \rho} a_{m}^{(k)}\right) \frac{\partial \rho}{\partial x_{m}}=0
\end{gathered}
$$

where $j=\overline{1, n}$ and $k \neq s=\overline{1,2}$, and whose solution is exactly the desired map (3.27). Inasmuch as we have only two functional parameters $b^{(s)}: \mathbb{R}^{n+1} \times \mathbb{R}^{2} \rightarrow \mathbb{R}, s=\overline{1,2}$, the system of $2 n$ differential relationships (3.32) can be, in general, compatible only for the case $n=1$. For all other cases $n \geq 2$ the compatibility condition for (3.32) must be checked separately by calculations.

\subsection{Example: the Gibbons-Tsarev equation}

As a first degenerate case of the scheme (3.19) above, we consider a compatible nonlinear first order system $\left.J^{1}\left(\mathbb{R}^{2} ; \mathbb{R}\right)\right|_{\mathscr{E}} \subset J^{1}\left(\mathbb{R}^{2} ; \mathbb{R}\right)$ at $n=0$, as discussed in [34]:

$$
\frac{\partial w}{\partial t}-\frac{1}{z_{y}+z_{t} w-w^{2}}=0, \frac{\partial w}{\partial y}+\frac{z_{t}-w}{u_{y}+z_{t} w-w^{2}}=0,
$$

first derived in [27], where $(t, y ; w) \in \mathbb{R}^{2} \times \mathbb{R}$ and a map $u: \mathbb{R}^{2} \rightarrow \mathbb{R}$ satisfies the Gibbon-Tsarev equation $\mathscr{E}[y, t ; u]=0$ in the form

$$
z_{y y}+z_{t} z_{t y}-z_{y} z_{t t}+1=0 .
$$

Since the nonlinear system (3.33) is compatible and the adjacent system of vector field flows (3.25) it follows that any solution $w: \mathbb{R}^{2} \rightarrow \mathbb{R}$ to (3.33) generates a first integral $\psi: \mathbb{R} \times \mathbb{R}^{2} \rightarrow \mathbb{R}$ of a system of equations

$$
\frac{\partial \psi}{\partial t}+\frac{1}{z_{y}+z_{t} \lambda-\lambda^{2}} \frac{\partial \psi}{\partial \lambda}=0, \frac{\partial \psi}{\partial y}-\frac{z_{t}-\lambda}{z_{y}+z_{t} \lambda-\lambda^{2}} \frac{\partial \psi}{\partial \lambda}=0
$$

where $\psi(\lambda ; y, t):=\alpha(w(t, y)-\lambda)$ for all $(\lambda ; t, y) \in \mathbb{R} \times \mathbb{R}^{2}$ and some smooth map $\alpha: \mathbb{R} \rightarrow \mathbb{R}$. The compatible system (3.35) considered as the jet-submanifold $\left.J_{\text {lin }}^{1}\left(\mathbb{R}^{1} \times \mathbb{R}^{2} ; \mathbb{R}\right)\right|_{\mathscr{E}} \subset J^{1}\left(\mathbb{R}^{1} \times \mathbb{R}^{2} ; \mathbb{R}\right)$ solves the problem of constructing the linearizing implication (3.19).

As was demonstrated in $[28,40]$, the substitution

$$
u:=\frac{1}{2}\left(-z_{t}+\sqrt{z_{t}^{2}+4 z_{y}}\right), v:=\frac{1}{2}\left(-z_{t}-\sqrt{z_{t}^{2}+4 z_{y}}\right)
$$

gives rise to the equivalent dynamical system

$$
u_{y}=v u_{t}-(u-v)^{-1}, \quad v_{y}=u v_{t}+(u-v)^{-1}
$$


on a functional space $M \subset C^{\infty}\left(\mathbb{R} ; \mathbb{R}^{2}\right)$ subject to the evolution parameter $y \in \mathbb{R}$ modulo evolution with respect to the joint evolution parameter $t \in \mathbb{R}$. Taking into account the Lax-Sato representation (3.35), one readily obtains the corresponding linearizing Lax-Sato representation for the dynamical system (3.37):

$$
\frac{\partial \psi}{\partial t}-\frac{1}{(\lambda+u)(\lambda+v)} \frac{\partial \psi}{\partial \lambda}=0, \frac{\partial \psi}{\partial y}-\frac{u+v+\lambda}{(\lambda+u)(\lambda+v)} \frac{\partial \psi}{\partial \lambda}=0 .
$$

The above Lax-Sato representation can be now reanalyzed more deeply within the Lie-algebraic scheme devised recently in [31]. Namely, we define the complex torus diffeomorphism Lie group $\tilde{G}:=\operatorname{Diff}\left(\mathbb{T}_{\mathbb{C}}^{1}\right)$, holomorphically extended in the interior $\mathbb{S}_{+}^{1} \subset \mathbb{C}$ and in the exterior $\mathbb{S}_{-}^{1} \subset \mathbb{C}$ regions of the unit circle $\mathbb{S}^{1} \subset \mathbb{C}^{1}$, such that for any $\left.g(\lambda) \in \tilde{G}\right|_{\mathbb{S}_{-}^{1}}, \lambda \in \mathbb{S}_{-}^{1}, g(\infty)=1 \in \operatorname{Diff}\left(\mathbb{T}^{1}\right)$, and study its specially chosen coadjoint orbits, related to the compatible system of linear vector field equations (3.38).

As a first step for solving this problem one needs to consider the corresponding Lie algebra $\tilde{\mathscr{G}}:=\operatorname{diff}\left(\mathbb{T}_{\mathbb{C}}^{1}\right)$ and its decomposition into the direct sum

$$
\tilde{\mathscr{G}}=\tilde{\mathscr{G}}_{+} \oplus \tilde{\mathscr{G}}_{-}
$$

of Laurent series with positive as $|\lambda| \rightarrow 0$ and strongly negative as $\lambda \mid \rightarrow \infty$ degrees, respectively. Then, it follows from Adler-Kostant-Symes theory, that for any element $\tilde{l} \in \tilde{\mathscr{G}}^{*} \simeq \Lambda^{1}\left(\mathbb{T}_{\mathbb{C}}^{1}\right)$ the following formally constructed flows

$$
d \tilde{l} / d y=-a d_{\nabla h^{(y)}(\tilde{l})}^{*} \tilde{l}, \quad d \tilde{l} / d t=-a d_{\nabla h^{(t)}(\tilde{l})}^{*} \tilde{l}
$$

along the evolution parameters $y, t \in \mathbb{R}^{2}$ are always compatible, if $h^{\left(p_{y}\right)}$ and $h^{\left(p_{t}\right)} \in I\left(\tilde{\mathscr{G}}^{*}\right)$ are arbitrarily chosen functionally independent Casimir functionals on the adjoint space $\tilde{\mathscr{G}}^{*}$ and $\nabla h^{(y)}(\tilde{l}):=\nabla h^{\left(p_{y}\right)}(\tilde{l})_{-}, \nabla h^{(t)}(\tilde{l}):=\nabla h^{\left(p_{t}\right)}(\tilde{l})_{-}$are their gradients, suitably projected on the subalgebra $\tilde{\mathscr{G}}_{-}$. Keeping in mind this result, consider the Casimir functional $h^{\left(p_{y}\right)}$ on $\tilde{\mathscr{G}}^{*}$, whose gradient $\nabla h^{\left(p_{y}\right)}(\tilde{l}):=\nabla h^{\left(p_{y}\right)}(l) \partial / \partial \lambda \in \tilde{\mathscr{G}}$ as $|\lambda| \rightarrow \infty$ is taken, for simplicity, in the asymptotic form

$$
\nabla h^{\left(p_{y}\right)}(\tilde{l}) \sim\left(\frac{\lambda+u+v}{(\lambda+u)(\lambda+v)}+\alpha_{0}+\alpha_{1} \lambda\right) \frac{\partial}{\partial \lambda},
$$

where $\lambda \in \mathbb{T}_{\mathbb{C}}^{1},|\lambda| \rightarrow \infty$, and the coefficients $\alpha_{j} \in C^{\infty}\left(\mathbb{R}^{2} ; \mathbb{R}\right), j=\overline{0,1}$, are arbitrarily chosen nontrivial functional parameters, giving rise to the gradient projection

$$
\nabla h^{(y)}(\tilde{l}):=\left.\nabla h^{\left(p_{y}\right)}(\tilde{l})\right|_{-}=\frac{\lambda+u+v}{(\lambda+u)(\lambda+v)},
$$

generating the first flow of (3.40). As the differential 1-form $\tilde{l}=l(\lambda ; y, t) d \lambda \in \Lambda^{1}\left(\mathbb{T}_{\mathbb{C}}^{1}\right) \simeq \tilde{\mathscr{G}}$ satisfies, by definition, the condition

$$
a d_{\nabla h^{(p y)}(\tilde{l})}^{*} \tilde{l}=0,
$$

equivalent to the differential equation

$$
\frac{d}{d \lambda}\left[l(\lambda ; y, t)\left(\nabla h^{\left(p_{y}\right)}(l)\right)^{2}\right]=0,
$$

one readily obtains from (3.42) and (3.44) the coefficient

$$
l(\lambda ; y, t)=\left(\nabla h^{\left(p_{y}\right)}(l)\right)^{-2}=\frac{(\lambda+u)^{2}(\lambda+v)^{2}}{\left[\lambda+u+v+\left(\alpha_{0}+\alpha_{1} \lambda\right)(\lambda+u)(\lambda+v)\right]^{2}},
$$

satisfying the relationship $l(\lambda ; y, t)\left(\nabla h^{\left(p_{y}\right)}(l)\right)^{2}=1$ for all $(t, y) \in \mathbb{R}^{2}$.

Now we prove the following useful observation.

Lemma 3.6. The set $\mathrm{I}\left(\tilde{\mathscr{G}}^{*}\right)$ of functionally independent Casimir invariants is one-dimensional.

Proof. Any asymptotic solution to the determining equation (3.47) satisfies the symmetry invariance with respect to the multiplication $\nabla h^{\left(p_{t}\right)}(\tilde{l}) \rightarrow \sigma(t, y) \nabla h^{\left(p_{t}\right)}(\tilde{l})$ by an arbitrary smooth function $\sigma: \mathbb{R}^{2} \rightarrow \mathbb{R}$, which proves the lemma.

Consider now the gradient $\nabla h^{\left(p_{t}\right)}(\tilde{l}) \in \tilde{\mathscr{G}}$ of the Casimir functional $h^{\left(p_{t}\right)} \in \mathrm{D}\left(\tilde{\mathscr{G}}^{*}\right)$, which satisfies, as does (3.43), the condition

$$
\left.a d_{\nabla h\left(p_{t}\right)}^{*} \tilde{l}\right)(=0,
$$

which is equivalent to the following linear differential equation

$$
2 l(\lambda ; y, t) \frac{\partial}{\partial \lambda} \nabla h^{\left(p_{t}\right)}(\tilde{l})+\nabla h^{\left(p_{t}\right)}(\tilde{l}) \frac{\partial}{\partial \lambda} l(\lambda ; y, t)=0 .
$$

Its solution can be naturally represented as the asymptotic series

$$
\nabla h^{\left(p_{t}\right)}(\tilde{l}) \sim \frac{1}{(\lambda+u)(\lambda+v)}+\sum_{j \in \mathbb{Z}_{+}} \beta_{j} \lambda^{j}
$$

for some nontrivial coefficients $\beta_{j} \in C^{\infty}\left(\mathbb{R}^{2} ; \mathbb{R}\right)$, successively determined from the equation (3.47).

From Lemma 3.6, we see that the solution (3.48) to the determining equation (3.47) is unique owing to its natural symmetry invariance with respect to the multiplication $\nabla h^{\left(p_{t}\right)}(\tilde{l}) \rightarrow \sigma(t, y) \nabla h^{\left(p_{t}\right)}(\tilde{l})$ by an arbitrary smooth function $\sigma: \mathbb{R}^{2} \rightarrow \mathbb{R}$. In particular, this means that 
asymptotically as $\lambda \rightarrow \infty$ the product $l(\lambda ; y, t)\left(\nabla h^{\left(p_{t}\right)}(l)\right)^{2} \sim 0$, for otherwise if $l(\lambda ; y, t)\left(\nabla h^{\left(p_{t}\right)}(l)\right)^{2} \nrightarrow 0$, this product becomes, owing to (3.44), a nonzero constant subject to the parameter $\lambda \in \mathbb{C}$. This means that $\nabla h^{\left(p_{t}\right)}(l)=\nabla h^{\left(p_{y}\right)}(\tilde{l}) \sigma(t, y)$ for any smooth arbitrary function $\sigma \in C^{\infty}\left(\mathbb{R}^{2} ; \mathbb{R}\right)$, producing no new flow with respect to the evolution parameter $t \in \mathbb{R}$.

Consider now the gradient projection

$$
\nabla h^{(t)}(\tilde{l}):=\left.\nabla h^{\left(p_{t}\right)}(\tilde{l})\right|_{-}=\frac{1}{(\lambda+u)(\lambda+v)}
$$

generating the second flow of (3.40). As a consequence of the results above we can easily derive the following compatibility condition for the flows (3.40):

$$
\left[\frac{\partial}{\partial y}-\nabla h^{(y)}(\tilde{l}), \frac{\partial}{\partial y}-\nabla h^{(t y)}(\tilde{l})\right]=0
$$

which is equivalent modulo the dynamical system (3.37) to the following system of two a priori compatible linear vector field equations:

$$
\frac{\partial \psi}{\partial y}-\frac{\lambda+u+v}{(\lambda+u)(\lambda+v)} \frac{\partial \psi}{\partial \lambda}=0, \quad \frac{\partial \psi}{\partial t}-\frac{1}{(\lambda+u)(\lambda+v)} \frac{\partial \psi}{\partial \lambda}=0
$$

for $\psi \in C^{2}(\mathbb{R} ; \mathbb{R})$ and all $(\lambda ; t, y) \in \mathbb{C} \times \mathbb{R}^{2}$. Thus, we can formulate the results, obtained above, as the following proposition.

Proposition 3.7. A system $\left.J_{\text {lin }}^{1}\left(\mathbb{R} \times \mathbb{R}^{2} ; \mathbb{R}\right)\right|_{\mathscr{E}}$ of the linear first order equations (3.51) on the covering jet-manifold $J^{1}\left(\mathbb{R} \times \mathbb{R}^{2} ; \mathbb{R}\right)$ is compatible; that is, it holds for any its smooth solution $\psi: \mathbb{R} \times \mathbb{R}^{2} \rightarrow \mathbb{R}$ at all points $(\lambda ; y, t) \in \mathbb{R} \times \mathbb{R}^{2}$ iff the function $u: \mathbb{R}^{2} \rightarrow \mathbb{R}$ satisfies the Gibbons-Tsarev equation (3.34).

Moreover, taking into account that the flows (3.40) are Hamiltonian systems on the coadjoint space $\tilde{\mathscr{G}}^{*}$, their reductions on the space of functional variables $(u, v) \in C^{\infty}\left(\mathbb{R}^{2} ; \mathbb{R}^{2}\right)$ are also Hamiltonian. This reduction scheme is now under study and shall be presented elsewhere.

\subsection{Example: the $\mathrm{ABC}$-equation}

As the second example we consider a compatible system $\left.J^{1}\left(\mathbb{R} \times \mathbb{R}^{2} ; \mathbb{R}\right)\right|_{\mathscr{E}}$ of the nonlinear first order differential equations (3.6) on the jet-manifold $J^{1}\left(\mathbb{R} \times \mathbb{R}^{2} ; \mathbb{R}\right)$. It is easy to check that the adjacent system of vector field flows

$$
\frac{\partial x}{\partial t}=\frac{u_{t} w}{u_{x} k_{1}\left(k_{1}+k_{2}-1\right)}, \quad \frac{\partial x}{\partial y}=\frac{u_{y} w}{u_{x} k_{1}\left(w+k_{1}+k_{2}-1\right)},
$$

modulo the relationships (3.6), is not compatible for all $(w ; t, y) \in \mathbb{R} \times \mathbb{R}^{2}$. Thus, we need to construct a map (3.23) such that the resulting system (3.24) will possess an adjacent system of vector field flows already compatible for all $(\tilde{w} ; t, y) \in \mathbb{R} \times \mathbb{R}^{2}$. To do this let us get rid of to begin with the strictly linear part of the equations (3.6), giving rise to the representation of its solution as $w(x ; t, y)=\left(u_{x}(x ; t, y)\right)^{\alpha} \tilde{w}(x ; t, y)$ for $\alpha=\left(k_{1}+k_{2}-1\right) / k_{1}$ and all $(x ; t, y) \in \mathbb{R} \times \mathbb{R}^{2}$. Its corresponding substitution into (3.6) yields the following a priori compatible system $\left.J^{1}\left(\mathbb{R} \times \mathbb{R}^{2} ; \mathbb{R}\right)\right|_{\mathscr{E}}$ of the first order equations

$$
\begin{gathered}
\frac{\partial \tilde{w}}{\partial t}+\frac{\tilde{w} u_{x}^{\alpha-1} u_{t}}{k_{1} \alpha} \frac{\partial \tilde{w}}{\partial x}+\frac{\tilde{w}^{2} u_{x}^{\alpha}\left(u_{t} u_{x x}-k_{1} u_{x x} u_{x t}\right)}{k_{1}^{2}}=0 \\
\frac{\partial \tilde{w}}{\partial y}+\frac{k_{2} \tilde{w} u_{x}^{\alpha-1} u_{y}}{k_{1}\left(\tilde{w} u_{x}^{\alpha}+\alpha\right)} \frac{\partial \tilde{w}}{\partial x}+\frac{k_{2} \alpha \tilde{w}^{2} u_{x}^{\alpha} u_{y} u_{x x}}{k_{1}^{2}\left(\tilde{w} u_{x}^{\alpha}+\alpha\right)}=0
\end{gathered}
$$

on the jet-manifold $J^{1}\left(\mathbb{R} \times \mathbb{R}^{2} ; \mathbb{R}\right)$. We can now easily check that the above expression $\tilde{w}(x ; t, y)=\left(u_{x}(x ; t, y)\right)^{-\alpha} w(x ; t, y)$ for all $(x ; t, y) \in \mathbb{R} \times \mathbb{R}^{2}$, determining the map (3.23), is exactly the one searched for, inasmuch the corresponding adjacent system of vector field flows

$$
\frac{\partial x}{\partial t}=\frac{\tilde{w} u_{x}^{\alpha-1} u_{t}}{k_{1} \alpha}, \frac{\partial x}{\partial y}=\frac{k_{2} \tilde{w} u_{x}^{\alpha-1} u_{y}}{k_{1}\left(\tilde{w} u_{x}^{\alpha}+\alpha\right)}
$$

on $\mathbb{R} \times \mathbb{R}^{2}$ proves to be compatible modulo the system $\left.\quad J^{1}\left(\mathbb{R} \times \mathbb{R}^{2} ; \mathbb{R}\right)\right|_{\mathscr{E}}$ of a priori compatible differential relationships (3.53) on $J^{1}\left(\mathbb{R} \times \mathbb{R}^{2} ; \mathbb{R}\right)$. Based on this compatibility result, one can easily construct the corresponding linearizing system $\left.J_{\text {lin }}^{1}\left(\mathbb{R}^{2} \times \mathbb{R}^{2} ; \mathbb{R}\right)\right|_{\mathscr{E}}$ on the covering jet-manifold $J^{1}\left(\mathbb{R}^{2} \times \mathbb{R}^{2} ; \mathbb{R}\right)$, realizing the inverse implication (3.19) as the Lax-Sato representation

$$
\begin{gathered}
\frac{\partial \psi}{\partial t}+\frac{\lambda u_{x}^{\alpha-1} u_{t}}{k_{1} \alpha} \frac{\partial \psi}{\partial x}-\frac{\lambda^{2} u_{x}^{\alpha}\left(u_{t} u_{x x}-k_{1} u_{x x} u_{x t}\right)}{k_{1}^{2}} \frac{\partial \psi}{\partial \lambda}=0, \\
\frac{\partial \psi}{\partial y}+\frac{k_{2} \lambda u_{x}^{\alpha-1} u_{y}}{k_{1}\left(\lambda u_{x}^{\alpha}+\alpha\right)} \frac{\partial \psi}{\partial x}-\frac{\lambda^{2} k_{2} \alpha u_{x}^{\alpha-1} u_{y} u_{x x}}{k_{1}^{2}\left(\lambda u_{x}^{\alpha}+\alpha\right)} \frac{\partial \psi}{\partial \lambda}=0
\end{gathered}
$$

exactly coinciding with (3.7), where $\psi(x, \lambda ; t, y):=\alpha(\tilde{w}(x ; t, y)-\lambda)$ for all $(x, \lambda ; t, y) \in \mathbb{R}^{2} \times \mathbb{R}^{2}$ and any smooth mapping $\alpha: \mathbb{R} \rightarrow \mathbb{R}$. Thus, the linear differential system (3.55) solves the above problem of constructing the inverse implication (3.19) for the compatible nonlinear differential system $\left.J^{1}\left(\mathbb{R} \times \mathbb{R}^{2} ; \mathbb{R}\right)\right|_{\mathscr{E}}$ (3.6), thereby proving Proposition 3.4. 


\subsection{Example: the Manakov-Santini equations}

The Manakov-Santini equations

$$
\begin{aligned}
u_{t x}+u_{y y}+\left(u u_{x}\right)_{x}+v_{x} u_{x y}-v_{y} u_{x x} & =0, \\
v_{x t}+v_{y y}+u v_{x x}+v_{x} v_{x y}-v_{y} v_{x x} & =0,
\end{aligned}
$$

where $(u, v) \in C^{\infty}\left(\mathbb{R} \times \mathbb{R}^{2} ; \mathbb{R}^{2}\right)$, as well known [39], are obtained as some generalization of the dispersionless reduction for the KadomtsevPetviashvili equation. It possesses the following compatible nonlinear first order differential covering $\left.J^{1}\left(\mathbb{R} \times \mathbb{R}^{2} ; \mathbb{R}\right)\right|_{\mathscr{E}} \subset J^{1}\left(\mathbb{R} \times \mathbb{R}^{2} ; \mathbb{R}\right)$ as

$$
\begin{gathered}
\frac{\partial w}{\partial t}+\left(w^{2}-w v_{x}+u-v_{y}\right) \frac{\partial w}{\partial x}+\left(w-v_{x}\right)\left(u_{x}-w v_{x x}\right)-u_{y}+v_{y y}+v_{x} v_{x y}=0 \\
\frac{\partial w}{\partial y}+w \frac{\partial w}{\partial x}-v_{x x} w+\left(u-v_{y}\right)_{x}=0
\end{gathered}
$$

giving rise for all $(x ; t, y) \in \mathbb{R} \times \mathbb{R}^{2}$ to the Manakov-Santini differential relationship $\mathscr{E}[x ; y, t ; u, v]=0(3.56)$ as a submanifold on the adjacent jet-manifold $J^{2}\left(\mathbb{R} \times \mathbb{R}^{2} ; \mathbb{R}^{2}\right)$. It is now easy to check that the naturally related to (3.57) system of vector field flows

$$
\frac{\partial x}{\partial t}=w^{2}-w v_{x}+u-v_{y}, \quad \frac{\partial x}{\partial y}=w
$$

is for all $(t, y) \in \mathbb{R}^{2}$ not compatible modulo these differential relationships (3.57). Thus, one needs to construct such an isomorphic transformation

$$
J^{1}\left(\mathbb{R}^{2} \times \mathbb{R}^{2} ; \mathbb{R}\right) \ni(x, w ; t, y) \rightarrow(x, \tilde{w} ; t, y) \in J^{1}\left(\mathbb{R}^{2} \times \mathbb{R}^{2} ; \mathbb{R}\right)
$$

that the expression $w(x ; y, t)=\beta(x, \tilde{w} ; y, t)$ for some, in general nonlinear, smooth mapping $\beta: \mathbb{R}^{2} \times \mathbb{R}^{2} \rightarrow \mathbb{R}$ transforms the first order differential covering (3.57) into an equivalent compatible first order differential covering (3.24), for which the corresponding vector field flows (3.25) become also compatible. This problem is easily enough solved, giving rise to the simple mapping:

$$
w=\tilde{w}+v_{x}
$$

from which one ensues the following compatible first order differential covering:

$$
\begin{gathered}
\frac{\partial \tilde{w}}{\partial t}+\left(\tilde{w}^{2}+\tilde{w} v_{x}+u-v_{y}\right) \frac{\partial \tilde{w}}{\partial x}-u_{y}+\tilde{w} u_{x}=0, \\
\frac{\partial \tilde{w}}{\partial y}+\left(v_{x}+\tilde{w}\right) \frac{\partial \tilde{w}}{\partial x}+u_{x}=0 .
\end{gathered}
$$

It is easy to check that the naturally related to (3.61) system of vector field flows

$$
\frac{\partial x}{\partial t}=\tilde{w}^{2}+\tilde{w} v_{x}+u-v_{y}, \quad \frac{\partial x}{\partial y}=v_{x}+\tilde{w}
$$

is already compatible for all $(t, y) \in \mathbb{R}^{2}$ modulo these differential relationships (3.61). Based on this compatibility result, stated above, one can easily construct the corresponding linearizing first order differential system $\left.J_{\text {lin }}^{1}\left(\mathbb{R}^{2} \times \mathbb{R}^{2} ; \mathbb{R}\right)\right|_{\mathscr{E}}$ on the covering jet-manifold $J^{1}\left(\mathbb{R}^{2} \times \mathbb{R}^{2} ; \mathbb{R}\right)$, realizing the inverse implication (3.19) as the Lax-Sato representation

$$
\begin{gathered}
\frac{\partial \psi}{\partial t}+\left(\lambda^{2}+\lambda v_{x}+u-v_{y}\right) \frac{\partial \psi}{\partial x}+\left(u_{y}-\lambda u_{x}\right) \frac{\partial \psi}{\partial \lambda}=0, \\
\frac{\partial \psi}{\partial y}+\left(v_{x}+\lambda\right) \frac{\partial \psi}{\partial x}-u_{x} \frac{\partial \psi}{\partial \lambda}=0
\end{gathered}
$$

thus proving proposition 3.3 .

\subsection{The contact geometry linearization covering scheme}

\subsection{Short setting}

We consider two Hamilton-Jacobi type compatible for all $(x ; \tau):=(x ; t, y) \in \mathbb{R} \times \mathbb{R}^{2}$ first order differential relationships:

$$
\frac{\partial z}{\partial t}+\tilde{H}^{(t)}(x, z, \partial z / \partial x ; t, y)=0, \frac{\partial z}{\partial y}+\tilde{H}^{(y)}(x, z, \partial z / \partial x ; t, y)=0
$$

where $z: \mathbb{R}^{3} \rightarrow \mathbb{R}$ is a so called "action function" and $\tilde{H}^{(t)}, \tilde{H}^{(y)}: \mathbb{R}^{3} \times \mathbb{R}^{2} \rightarrow \mathbb{R}$ are smooth generalized Hamiltonian functions. The relationships 3.64 follow from the contact geometry $[12,19]$ interpretation of some mechanical systems, generated by vector fields. Namely, a differential one-form $\alpha^{(1)} \in \Lambda^{1}\left(\mathbb{R}^{3} \times \mathbb{R}\right)$, defined by the expression

$$
\alpha^{(1)}:=d z-\lambda d x
$$


is called contact and vector fields $X_{H^{(t)}}, X_{H^{(y)}} \in \Gamma\left(T\left(\mathbb{R}^{3} \times \mathbb{R}\right)\right)$ are called contact vector fields, if there exist functions $\mu^{(t)}, \mu^{(y)}: \mathbb{R}^{3} \times \mathbb{R} \rightarrow \mathbb{R}$, such that for all $(x, z ; \tau) \in \mathbb{R}^{2} \times \mathbb{R}^{2}$ the following equalities

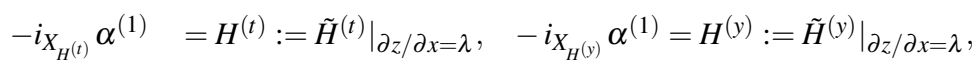

$$
\begin{aligned}
& \mathscr{L}_{H^{(t)}} \alpha^{(t)} \quad=\mu^{(t)} \alpha^{(t)}, \quad \mathscr{L}_{H^{(y)}} \alpha^{(y)}=\mu^{(y)} \alpha^{(y)}
\end{aligned}
$$

hold, where $\mathscr{L}_{H^{(t)}}, \mathscr{L}_{H^{(y)}}$ are the corresponding Lie derivatives [17, 29, 19] with respect to the vector fields $X_{H^{(t)}}, X_{H^{(y)}} \in \Gamma\left(T\left(\mathbb{R}^{3} \times \mathbb{R}\right)\right)$. From the conditions (3.66) one finds $[32,58]$ easily that

$$
\begin{aligned}
& X_{H^{(t)}}=\frac{\partial H^{(t)}}{\partial \lambda} \frac{\partial}{\partial x}-\left(\frac{\partial H^{(t)}}{\partial x}+\lambda \frac{\partial H^{(t)}}{\partial z}\right) \frac{\partial}{\partial \lambda}+\left(-H^{(t)}+\lambda \frac{\partial H^{(t)}}{\partial \lambda}\right) \frac{\partial}{\partial z}, \\
& X_{H^{(y)}}=\frac{\partial H^{(y)}}{\partial \lambda} \frac{\partial}{\partial x}-\left(\frac{\partial H^{(y)}}{\partial x}+\lambda \frac{\partial H^{(y)}}{\partial z}\right) \frac{\partial}{\partial \lambda}+\left(-H^{(y)}+\lambda \frac{\partial H^{(y)}}{\partial \lambda}\right) \frac{\partial}{\partial z},
\end{aligned}
$$

where $H^{(t)}:=\left.\tilde{H}^{(t)}\right|_{\partial z / \partial x=\lambda}, H^{(y)}:=\left.\tilde{H}^{(y)}\right|_{\partial z / \partial x=\lambda}$, and the compatibility of the nonlinear relationships (3.64) is equivalent to the commutativity of the following vector fields:

$$
\left[\frac{\partial}{\partial t}+X_{H^{(t)}}, \frac{\partial}{\partial y}+X_{H^{(y)}}\right]=0
$$

for all $(x, z, \lambda ; \tau) \in \mathbb{R}^{3} \times \mathbb{R}^{2}$, depending parametrically on $\lambda \in \mathbb{R}$. The latter can be rewritten as a compatible Lax-Sato representation for the vector field equations

$$
\frac{\partial \psi}{\partial t}+X_{H^{(t)}} \psi=0, \frac{\partial \psi}{\partial y}+X_{H^{(y)}} \psi=0
$$

for smooth invariant functions $\psi \in C^{2}\left(\mathbb{R}^{3} \times \mathbb{R}^{2} ; \mathbb{R}\right)$ and all $(x, z, \lambda ; \tau) \in \mathbb{R}^{3} \times \mathbb{R}^{2}$.

Remark 3.8. It is worth mentioning that when the Hamiltonian functions in (3.64) do not depend on the "action function" $z: \mathbb{R}^{3} \rightarrow \mathbb{R}$, the contact vector fields naturally reduce to the classical Hamiltonian ones:

$$
X_{H^{(t)}}=\frac{\partial H^{(t)}}{\partial \lambda} \frac{\partial}{\partial x}-\frac{\partial H^{(t)}}{\partial x} \frac{\partial}{\partial \lambda}, \quad X_{H^{(y)}}=\frac{\partial H^{(y)}}{\partial \lambda} \frac{\partial}{\partial x}-\frac{\partial H^{(y)}}{\partial x} \frac{\partial}{\partial \lambda}
$$

well known [19] from symplectic geometry.

\subsection{Example: the differential Toda singular manifold equation}

Some examples of applying this contact geometry linearization scheme to integrable $3 D$-dispersionless equations were recently presented by A. Sergyeyev [71]. We shall apply this scheme to a degenerate case when the system (3.64) is given by the following linear equations

$$
\frac{\partial z}{\partial t}+\frac{\left(e^{-2 u_{t}}-1\right)}{2 u_{x}} \frac{\partial z}{\partial x}=0, \frac{\partial z}{\partial y}-u_{y} u_{x}^{-1} e^{-2 u_{t}} \frac{\partial z}{\partial x}=0
$$

for a smooth map $z: \mathbb{R} \times \mathbb{R}^{2} \rightarrow \mathbb{R}$, whose compatibility condition is the interesting [16] differential Toda singular manifold equation (3.12) on a smooth function $u: \mathbb{R} \times \mathbb{R}^{2} \rightarrow \mathbb{R}$ for all $(x ; y, t) \in \mathbb{R} \times \mathbb{R}^{2}$, which defines a differential relationship $\left.J^{2}\left(\mathbb{R}^{2} \times \mathbb{R}^{2} ; \mathbb{R}\right)\right|_{\mathscr{E}} \subset J^{2}\left(\mathbb{R}^{2} \times \mathbb{R}^{2} ; \mathbb{R}\right)$ on the jet-manifold $J^{2}\left(\mathbb{R}^{2} \times \mathbb{R}^{2} ; \mathbb{R}\right)$

$$
u_{x y} s h^{2} u_{t}=u_{x} u_{y} u_{t t}
$$

Even though the equations (3.71) are linear, they contain no "spectral" parameter $\lambda \in \mathbb{R}$ subject to which one can construct the related conservation laws for (3.17) and apply the modified inverse scattering transform to construct exact special solutions.

Nevertheless, the above contact geometry linearization scheme makes it possible present the system as a set of compatible Hamilton-Jacobi equations

$$
\frac{\partial z}{\partial t}+\frac{\left(e^{-2 u_{t}}-1\right)}{2 u_{x}} \lambda=0, \frac{\partial z}{\partial y}-\frac{u_{y} e^{-2 u_{t}}}{u_{x}} \lambda=0
$$

with the contact Hamiltonians

$$
H^{(t)}:=\frac{\left(e^{-2 u_{t}}-1\right)}{2 u_{x}} \lambda, \quad H^{(y)}:=-\frac{u_{y} e^{-2 u_{t}}}{u_{x}} \lambda,
$$

where the function $u: \mathbb{R}^{2} \times \mathbb{R}^{2} \rightarrow \mathbb{R}$ depends here on the additional variable $z \in \mathbb{R}$. Taking into account (3.67), one can construct the corresponding extended contact vector fields

$$
\begin{aligned}
& \tilde{X}_{H^{(t)}}:=\frac{\partial}{\partial t}+X_{H^{(t)}}=\frac{\partial}{\partial t}+\frac{\left(e^{-2 u_{t}}-1\right)}{2 u_{x}} \frac{\partial}{\partial x}-\left[\lambda\left(\frac{e^{-2 u_{t}}-1}{2 u_{x}}\right)_{x}+\lambda^{2}\left(\frac{e^{-2 u_{t}}-1}{2 u_{x}}\right)_{z}\right] \frac{\partial}{\partial \lambda}, \\
& \tilde{X}_{H^{(y)}} \quad:=\frac{\partial}{\partial y}+X_{H^{(y)}}=\frac{\partial}{\partial y}-\frac{u_{y} e^{-2 u_{t}}}{u_{x}} \frac{\partial}{\partial x}+\left[\lambda\left(\frac{u_{y} e^{-2 u_{t}}}{u_{x}}\right)_{x}+\lambda^{2}\left(\frac{u_{y} e^{-2 u_{t}}}{u_{x}}\right)_{z}\right] \frac{\partial}{\partial \lambda}
\end{aligned}
$$

compatible on the solution set to the equation (3.72) on $J^{2}\left(\mathbb{R}^{2} \times \mathbb{R}^{2} ; \mathbb{R}\right)$. This makes it possible to verify our final proposition on the contact geometry linearization covering of the system (3.72). 
Proposition 3.9. The linear first order differential equation (3.71) on $J^{2}\left(\mathbb{R}^{2} \times \mathbb{R}^{2} ; \mathbb{R}\right)$ allows the following, dual quadratic in the parameter $\lambda \in \mathbb{R}$, Lax-Sato linearization covering

$$
\begin{array}{r}
\frac{\partial \psi}{\partial t}+\frac{\left(e^{-2 u_{t}}-1\right)}{2 u_{x}} \frac{\partial \psi}{\partial x}-\left[\lambda\left(\frac{e^{-2 u_{t}}-1}{2 u_{x}}\right)_{x}+\lambda^{2}\left(\frac{e^{-2 u_{t}}-1}{2 u_{x}}\right)_{z}\right] \frac{\partial \psi}{\partial \lambda}=0 \\
\frac{\partial \psi}{\partial y}-\frac{u_{y} e^{-2 u_{t}}}{u_{x}} \frac{\partial \psi}{\partial x}+\left[\lambda\left(\frac{u_{y} e^{-2 u_{t}}}{u_{x}}\right)_{x}+\lambda^{2}\left(\frac{u_{y} e^{-2 u_{t}}}{u_{x}}\right)_{z}\right] \frac{\partial \psi}{\partial \lambda}=0
\end{array}
$$

for smooth invariant functions $\psi \in C^{2}\left(\mathbb{R}^{3} \times \mathbb{R}^{2} ; \mathbb{R}\right)$, all $(x, z, \lambda ; \tau) \in \mathbb{R}^{3} \times \mathbb{R}^{2}$ and any smooth solution $u: \mathbb{R}^{2} \times \mathbb{R}^{2} \rightarrow \mathbb{R}$ to the differential equation (3.72).

\section{Integrable heavenly type superflows and their Lie-algebraic structure}

\subsection{Introductory notions and notations}

We begin from preliminaries for the superconformal loop diffeomorphism groups and their superconformal loop algebras. Let $\tilde{G}$ be the superconformal group of smooth loops $\left\{\mathbb{C} \supset \mathbb{S}^{1} \rightarrow G\right\}$, where $G:=\operatorname{Diff}\left(\mathbb{T}^{1 \mid N}\right)$ is the group of superconformal diffeomorphisms of the $1 \mid N$-dimensional supertorus $\mathbb{T}^{1 \mid N}, N \in \mathbb{N}$, with coordinates $(x, \vartheta) \in \mathbb{T}^{1 \mid N} \simeq \Lambda_{0} \times \Lambda_{1}^{N}$ from the infinite-dimensional Grassmann algebra $\Lambda=\Lambda_{0} \oplus \Lambda_{1}$. A superconformal vector field is

$$
\tilde{a}:=a \frac{\partial}{\partial x}+\frac{1}{2}<D a, D>
$$

where $D:=\left(D_{\vartheta_{1}}, D_{\vartheta_{2}}, \cdots, D_{\vartheta_{N}}\right)^{\top}, D_{\vartheta_{j}}:=\frac{\partial}{\partial \vartheta_{j}}+\vartheta_{j} \frac{\partial}{\partial x}, j=\overline{1, N}, \vartheta:=\left(\vartheta_{1}, \ldots, \vartheta_{N}\right)^{\top}, a \in C^{\infty}\left(\mathbb{T}^{1 \mid N} ; \Lambda_{0}\right)$, and is defined by the condition that the Lie superderivation $\mathscr{L}_{\tilde{a}}: \Lambda^{1}\left(\mathbb{T}^{1 \mid N}\right) \rightarrow \Lambda^{1}\left(\mathbb{T}^{1 \mid N}\right)$ of the superdifferential 1-form

$$
\omega^{(1)}=d x+\sum_{j=1}^{N} \vartheta_{j} d \vartheta_{j}
$$

is conformal, that is

$$
\mathscr{L}_{\tilde{a}} \omega^{(1)}=\mu_{\tilde{a}} \omega^{(1)}
$$

for some $\mu_{\tilde{a}} \in C^{\infty}\left(\mathbb{T}^{1 \mid N} ; \Lambda_{0}\right)$. As a simple consequence of the condition (4.3), one also has the commutator of any two vector fields $\tilde{a}, \tilde{b} \in \tilde{\mathscr{G}}$ :

$$
\begin{aligned}
& {[\tilde{a}, \tilde{b}]:=\tilde{c}=c \frac{\partial}{\partial x}+\frac{1}{2}\langle D c, D>,} \\
& c=a \frac{\partial b}{\partial x}-b \frac{\partial a}{\partial x}+\frac{1}{2}\langle D a, D b>
\end{aligned}
$$

verifying that the set $\tilde{\mathscr{G}}:=\widetilde{\operatorname{dif} f}\left(\mathbb{T}^{1 \mid N}\right)$ of loop superconformal vector fields is a Lie algebra. One can naturally identify this loop Lie algebra $\tilde{\mathscr{G}}$ with a dense subspace of the dual space $\tilde{\mathscr{G}}^{*}$ through the parity

$$
(\tilde{l}, \tilde{a})_{0}:=\underset{\lambda \in \mathbb{C}}{\operatorname{res}}(l, a)_{H}
$$

where $\tilde{l}:=l d x \in \tilde{\mathscr{G}}^{*}$.

For $p \in \mathbb{Z}$ and for any superconformal vector field $\tilde{a} \in \tilde{\mathscr{G}}$ and element $\tilde{l} \in \tilde{\mathscr{G}}^{*}$

$$
\tilde{a}:=a \frac{\partial}{\partial x}+\frac{1}{2}<D a, D>, \quad \tilde{l}:=l d x,
$$

and the bilinear form

$$
(l, a)_{H}:=\int_{\mathbb{T}^{1 \mid N}} l(x, \vartheta) a(x, \vartheta) d x d^{N} \vartheta
$$

is determined by means of the integration with respect to the Berezin measures

$$
\int_{\mathbb{T}^{1 \mid 1}} \alpha(x) d x d \vartheta_{j}=0, \int_{\mathbb{T}^{1} \mid 1} \alpha(x) d x \vartheta_{j} d \vartheta_{j}=\int_{\mathbb{T}^{1}} \alpha(x) d x,
$$

where $\alpha \in C^{\infty}\left(\mathbb{T}^{1} ; \mathbb{R}\right), j \in \overline{1, N}$. There are two cases: the first one when $N=2 k-1, a \in C^{\infty}\left(\mathbb{T}^{1 \mid(2 k-1)} ; \Lambda_{0}\right), l \in C^{\infty}\left(\mathbb{T}^{1 \mid(2 k-1)} ; \Lambda_{1}\right)$ and the second one, when $N=2 k, a \in C^{\infty}\left(\mathbb{T}^{1 \mid 2 k} ; \Lambda_{0}\right), l \in C^{\infty}\left(\mathbb{T}^{1 \mid 2 k} ; \Lambda_{0}\right)$, where $k \in \mathbb{N}$.

The constructed loop Lie algebra $\mathscr{G}^{*}$ of superconformal vector fields on the supertorus $\mathbb{T}^{1 \mid N}$ allows the following natural splitting

$$
\tilde{\mathscr{G}}=\tilde{\mathscr{G}}_{+} \oplus \tilde{\mathscr{G}}_{-},
$$


where $\tilde{\mathscr{G}}_{+}$and $\tilde{\mathscr{G}}_{-}$are also loop Lie algebras, that is

$$
\left[\tilde{\mathscr{G}}_{+}, \tilde{\mathscr{G}}_{+}\right] \subset \tilde{\mathscr{G}}_{+}, \quad\left[\tilde{\mathscr{G}}_{-}, \tilde{\mathscr{G}}_{-}\right] \subset \tilde{\mathscr{G}}_{-} .
$$

This fact makes it possible to apply to the Lie algebra $\tilde{\mathscr{G}}$ the known Lie algebraic AKS-scheme of constructing integrable Hamiltonian flows on the coadjoint space $\tilde{\mathscr{G}}^{*}$. Namely, let a $\mathscr{R}$-structure endomorphism $\mathscr{R}: \tilde{\mathscr{G}} \rightarrow \tilde{\mathscr{G}}$ be defined as

$$
\mathscr{R}=\left(P_{+}-P_{-}\right) / 2
$$

where, by definition, the projections

$$
P_{ \pm} \tilde{\mathscr{G}}:=\tilde{\mathscr{G}}_{ \pm} \subset \tilde{\mathscr{G}}
$$

Then the following commutator

$$
[\tilde{a}, \tilde{b}]_{\mathscr{R}}:=[\mathscr{R} \tilde{a}, \tilde{b}]+[\tilde{a}, \mathscr{R} \tilde{b}]
$$

where $\tilde{a}, \tilde{b} \in \tilde{\mathscr{G}}$ defines, on the linear space $\tilde{\mathscr{G}}$ a new Lie structure, satisfying the Jacobi identity, and generating the deformed Lie-Poisson bracket

$$
\{f, g\}_{\mathscr{R}}:=\left(\tilde{l},[\nabla f(\tilde{l}), \nabla g(\tilde{l})]_{\mathscr{R}}\right),
$$

where $f, g \in \mathrm{D}\left(\tilde{\mathscr{G}}^{*}\right)$. The corresponding set of Casimir invariants $\mathrm{I}\left(\tilde{\mathscr{G}}^{*}\right)$ is generated by the functional $h \in \mathrm{D}\left(\tilde{\mathscr{G}}^{*}\right)$, satisfying

$$
a d_{\nabla h(\tilde{l})}^{*} \tilde{l}=0,
$$

where

$$
a d_{a}^{*} l=\partial l / \partial x a+\frac{4-N}{2} l \partial a / \partial x+\frac{(-1)^{N+1}}{2}<D l, D a>
$$

for any superconformal vector field $\tilde{a} \in \tilde{\mathscr{G}}$ and a fixed element $\tilde{l} \in \tilde{\mathscr{G}}^{*}$. Here

$$
\tilde{a}=a \frac{\partial}{\partial x}+\frac{1}{2}<D a, D>, \quad \tilde{l}=l d x
$$

The Adler-Kostant-Symes theorem allows us to construct an infinite hierarchy of mutually commuting Hamiltonian flows

$$
d \tilde{l} / d t_{p}=-a d_{\nabla h^{(p)}(\tilde{l})_{+}}^{*} \tilde{l},
$$

where $\nabla h^{(p)}(l)=\lambda^{p} \nabla h(l), t_{p} \in \mathbb{R}, \quad p \in \mathbb{Z}_{+}$, by means of the asymptotic as $|\lambda| \rightarrow \infty$ expansion

$$
\nabla h(l) \sim \sum_{j \in \mathbb{Z}_{+}} \nabla h_{j}(x, \vartheta) \lambda^{-j}
$$

for the gradient of a generating functional $h \in \mathscr{D}\left(\tilde{\mathscr{G}}^{*}\right)$. The evolution equations (4.17) take the following forms

$$
\begin{aligned}
d l / d t_{p}=( & -\nabla h^{(p)}(l)_{+} \frac{\partial}{\partial x}+\frac{1}{2}<D \nabla h^{(p)}(l)_{+}, D>+ \\
& \left.+\left(2-\frac{N}{2}\right) \frac{\partial \nabla h^{(p)}(l)_{+}}{\partial x}\right) l= \\
= & -\left(\tilde{A}_{\nabla h^{(p)}(l)_{+}}+B_{\nabla h^{(p)}(l)_{+}}\right) l
\end{aligned}
$$

where

$$
\tilde{A}_{\nabla h^{(p)}(\tilde{l})_{+}}:=\left(\nabla h^{(p)}(l)_{+} \frac{\partial}{\partial x}+\frac{1}{2}<D \nabla h^{(p)}(l)_{+}, D>\right.
$$

for all $p \in \mathbb{Z}_{+}$. Thus, as the flows (4.19) are commuting, the following proposition automatically holds.

Proposition 4.1. The commuting condition for any two flows $d / d t_{p_{1}}$ and $d / d t_{p_{2}}, p_{1} \neq p_{2} \in \mathbb{Z}_{+}$, from the hierarchy (4.17) is equivalent to the equality

$$
\frac{\partial}{\partial t_{p_{2}}} \tilde{A}_{\nabla h^{\left(p_{1}\right)}(\tilde{l})_{+}}-\frac{\partial}{\partial t_{p_{1}}} \tilde{A}_{\nabla h^{\left(p_{2}\right)}(\tilde{l})_{+}}=\left[\tilde{A}_{\nabla h^{\left(p_{1}\right)}(\tilde{l})_{+}}, \tilde{A}_{\nabla h^{\left(p_{1}\right)}(\tilde{l})_{+}}\right]
$$

or

$$
\frac{\partial}{\partial t_{p_{2}}} \nabla h^{\left(p_{1}\right)}(\tilde{l})_{+}-\frac{\partial}{\partial t_{p_{1}}} \nabla h^{\left(p_{2}\right)}(\tilde{l})_{+}=\left[\nabla h^{\left(p_{1}\right)}(\tilde{l})_{+}, \nabla h^{\left(p_{2}\right)}(\tilde{l})_{+}\right]
$$

Moreover, the relationship (4.22) is a compatibility condition for the first order partial differential equations

$$
\left(\frac{\partial}{\partial t_{p_{1}}}+\nabla h^{\left(p_{1}\right)}(\tilde{l})_{+}\right) \psi=0,\left(\frac{\partial}{\partial t_{p_{2}}}+\nabla h^{\left(p_{2}\right)}(\tilde{l})_{+}\right) \psi=0,
$$

where $\psi \in C^{2}\left(\mathbb{R}^{2} \times \mathbb{T}^{1 \mid N} ; \Lambda_{0}\right)$.

The procedure for reducing the relationship (4.22) on the corresponding coadjoint action orbits for different $p_{1}$ and $p_{2} \in \mathbb{Z}_{+}$allows us to obtain integrable superanalogs of known integrable two-dimensional systems of heavenly equations with the Lax-Sato representations in the forms (4.21). 


\subsection{Example: The superanalogs of the generalized Mikhalev-Pavlov equations and their Lax-Sato integrability}

We now consider the well-known Mikhalev-Pavlov [43, 48] heavenly equation

$$
u_{x t}+u_{y y}=u_{y} u_{x x}-u_{x} u_{x y}
$$

where $u \in C^{\infty}\left(\mathbb{R}^{2} \times \mathbb{T}^{1} ; \mathbb{R}\right)$ and $(t, y ; x) \in \mathbb{R}^{2} \times \mathbb{T}^{1}$, which is the compatibility condition for the vector fields

$$
\frac{\partial \psi}{\partial t}+\left(\lambda^{2}+\lambda u_{x}-u_{y}\right) \frac{\partial \psi}{\partial x}=0, \quad \frac{\partial \psi}{\partial y}+\left(\lambda+u_{y}\right) \frac{\partial \psi}{\partial x}=0
$$

for a function $\psi \in C^{\infty}\left(\mathbb{R}^{2} \times \mathbb{T}^{1} ; \mathbb{R}\right)$ and arbitrary evolution parameters $y, t \in \mathbb{R}$ and construct its Lax-Sato integrable superanalogs for different $N \in \mathbb{N}$.

When $N \in \mathbb{Z}_{+}$is odd, $N \neq 5$ and $l=\vartheta_{N}\left((N-5) u_{x} / 2+\lambda\right)-\xi / 2, u=u\left(x, \vartheta_{1}, \ldots, \vartheta_{N-1}\right), \xi=\xi\left(x, \vartheta_{1}, \ldots, \vartheta_{N-1}\right)$, the asymptotic expansion for the gradient of the generating functional $h \in \mathrm{D}\left(\tilde{\mathscr{G}}^{*}\right)$ as $|\lambda| \rightarrow \infty$ has the form

$$
\nabla h(l) \sim 1+\left(u_{x}+\vartheta_{N} \xi_{x}\right) \lambda^{-1}-\left(u_{y}+\vartheta_{N} \xi_{y}\right) \lambda^{-2}+\ldots
$$

If $p_{1}=1$ and $p_{2}=2$, one obtains from the equality (4.22) the system

$$
\begin{gathered}
u_{x t}+u_{y y}=u_{y} u_{x x}-u_{x} u_{y x}-\xi_{x} \xi_{y} / 2-\sum_{i=1}^{N-1}\left(D_{\vartheta_{i}} u_{x}\right)\left(D_{\vartheta_{i}} u_{y}\right) / 2, \\
\xi_{x t}+\xi_{y y}=u_{y} \xi_{x x}-u_{x} \xi_{y x}+u_{x x} \xi_{y} / 2-u_{y x} \xi_{x} / 2+ \\
+\sum_{i=1}^{N-1}\left(D_{\vartheta_{i}} u_{y}\right)\left(D_{\vartheta_{i}} \xi_{x}\right) / 2-\sum_{i=1}^{N-1}\left(D_{\vartheta_{i}} u_{x}\right)\left(D_{\vartheta_{i}} \xi_{y}\right) / 2
\end{gathered}
$$

where $t_{p_{1}}:=y, t_{p_{2}}:=t$ and $(u, \xi)^{\top} \in C^{\infty}\left(\mathbb{R}^{2} \times \mathbb{T}^{1 \mid(N-1)} ; \Lambda_{0} \times \Lambda_{1}\right)$. The compatibility condition for the first order partial differential equations such as

$$
\begin{gathered}
\psi_{y}+\left(\left(\lambda+u_{x}\right)+\vartheta_{N} \xi_{x}\right) \psi_{x}+ \\
+\sum_{i=1}^{N-1}\left(\left(D_{\vartheta_{i}} u_{x}\right)-\vartheta_{N}\left(D_{\vartheta_{i}} \xi_{x}\right)\right)\left(D_{\vartheta_{i}} \psi\right) / 2+ \\
+\left(\xi_{x}+\vartheta_{N} u_{x x}\right)\left(D_{\vartheta_{N}} \psi\right) / 2=0, \\
\frac{\partial \psi}{\partial t}+\left[\left(\lambda^{2}+u_{x} \lambda-u_{y}\right)+\vartheta_{N}\left(\xi_{x} \lambda-\xi_{y}\right)\right] \frac{\partial \psi}{\partial x}+ \\
+\sum_{i=1}^{N-1}\left(\left(\left(D_{\vartheta_{i}} u_{x}\right) \lambda-\left(D_{\vartheta_{i}} u_{y}\right)\right)-\right. \\
\left.-\vartheta_{N}\left(\left(D_{\vartheta_{i}} \xi_{x}\right) \lambda-\left(D_{\vartheta_{i}} \xi_{y}\right)\right)\right)\left(D_{\vartheta_{i}} \psi\right) / 2+ \\
+\left(\left(\xi_{x} \lambda-\xi_{y}\right)+\vartheta_{N}\left(u_{x x} \lambda-u_{y x}\right)\right)\left(D_{\vartheta_{N}} \psi\right) / 2=0,
\end{gathered}
$$

where $\psi \in C^{2}\left(\mathbb{R}^{2} \times \mathbb{T}^{1 \mid N} ; \Lambda_{0}\right)$, are Lax-Sato representation for the system (4.27).

When $N \in \mathbb{Z}_{+}$is even, $N \neq 4$ and $l=\left((N-4) u_{x} / 2+\lambda\right)+\vartheta_{N}(N-4) \xi_{x} / 2, u=u\left(x, \vartheta_{1}, \ldots, \vartheta_{N-1}\right), \xi=\xi\left(x, \vartheta_{1}, \ldots, \vartheta_{N-1}\right)$, the asymptotic expansion for the gradient of the generating functional $h \in \mathscr{D}\left(\tilde{\mathscr{G}}^{*}\right)$ as $|\lambda| \rightarrow \infty$ also has the form (4.26). For $p_{1}=1$ and $p_{2}=2$ one obtains from (4.22) the system (4.27), in which $t_{p_{1}}:=y, t_{p_{2}}:=t$ and $(u, \xi)^{\top} \in C^{\infty}\left(\mathbb{R}^{2} \times \mathbb{T}^{1 \mid(N-1)} ; \Lambda_{0} \times \Lambda_{1}\right)$.

The system (4.27) can be considered as a superconformal analog of the Mikhalev-Pavlov heavenly equation for every $N \in N \backslash\{4 ; 5\}$. Namely, for $N=1$ and $N=2$ one obtains easily the systems

$$
\begin{gathered}
u_{x t}+u_{y y}=u_{y} u_{x x}-u_{x} u_{y x}-\xi_{x} \xi_{y} / 2 \\
\xi_{x t}+\xi_{y y}=u_{y} \xi_{x x}-u_{x} \xi_{y x}+u_{x x} \xi_{y} / 2-u_{y x} \xi_{x} / 2
\end{gathered}
$$

and

$$
\begin{gathered}
u_{x t}+u_{y y}=u_{y} u_{x x}-u_{x} u_{y x}-\xi_{x} \xi_{y} / 2-\left(D_{\vartheta_{1}} u_{x}\right)\left(D_{\vartheta_{1}} u_{y}\right) / 2 \\
\xi_{x t}+\xi_{y y}=u_{y} \xi_{x x}-u_{x} \xi_{y x}+u_{x x} \xi_{y} / 2-u_{y x} \xi_{x} / 2+ \\
+\left(D_{\vartheta_{1}} u_{y}\right)\left(D_{\vartheta_{1}} \xi_{x}\right) / 2-\left(D_{\vartheta_{1}} u_{x}\right)\left(D_{\vartheta_{1}} \xi_{y}\right) / 2
\end{gathered}
$$

respectively.

It should be noted that for $N=4$ and $N=5$ one cannot find the asymptotic expansions for gradients of the generating functional $h \in \mathrm{D}\left(\tilde{\mathscr{G}}^{*}\right)$ as $|\lambda| \rightarrow \infty$ by means of the relationship (4.14) and as a consequence construct integrable superanalogs in the framework of the proposed Lie-algebraic approach.

\subsection{Example: The superanalogs of the generalized Liouville equations and their Lax-Sato integrability}

We now show using the Lie superalgebra $\tilde{\mathscr{G}}_{(1 \mid N)}:=\widetilde{\operatorname{dif} f}\left(\mathbb{T}_{\mathbb{C}}^{1 \mid N}\right)$ of the superconformal vector fields on $\mathbb{T}_{\mathbb{C}}^{1 \mid N} \simeq \mathbb{T}_{\mathbb{C}}^{1} \times \Lambda_{1}^{N}$, that the Lax-Sato integrable superanalogs of the Liouville heavenly equations can be obtained as a result of a diffeomorphism in the space of variables $(z, \vartheta) \in \mathbb{T}_{\mathbb{C}}^{1 \mid N}$

First one introduces the superderivatives $D_{\vartheta_{j}}:=\partial / \partial \vartheta_{j}+\vartheta_{j} \partial / \partial z, z \in \mathbb{T}_{\mathbb{C}}^{1}, \vartheta_{j} \in \Lambda_{1}, j=\overline{1, N}$, in the superspace $\Lambda_{0} \times \Lambda_{1}^{N}$. The loop Lie algebra $\tilde{\mathscr{G}}_{(1 \mid N)}$ is generated by the superconformal vector fields

$$
\tilde{a}:=a \frac{\partial}{\partial z}+\frac{1}{2}<D a, D>,
$$


where $D:=\left(D_{\vartheta_{1}}, D_{\vartheta_{2}}, \cdots, D_{\vartheta_{N}}\right)^{\top}, i=\overline{1, N}, \vartheta:=\left(\vartheta_{1}, \ldots, \vartheta_{N}\right)^{\top}, a \in C^{\infty}\left(\mathbb{T}_{\mathbb{C}}^{1 \mid N} ; \Lambda_{0}\right)$, with the commutator

$$
\begin{aligned}
& {[\tilde{a}, \bar{b}]:=\bar{c}=c \frac{\partial}{\partial z}+\frac{1}{2}\langle D c, D\rangle,} \\
& c=a \frac{\partial b}{\partial z}-b \frac{\partial a}{\partial z}+\frac{1}{2}\langle D a, D b>
\end{aligned}
$$

This loop Lie algebra allows the following splitting:

$$
\tilde{\mathscr{G}}_{(1 \mid N)}=\tilde{\mathscr{G}}_{(1 \mid N)+} \oplus \tilde{\mathscr{G}}_{(1 \mid N)-} .
$$

A nontrivial Casimir invariant $h^{\left(p_{y}\right)} \in \mathrm{I}\left(\tilde{\mathscr{G}}_{(1 \mid N)}^{*}\right)$ satisfies the relationship

$$
\left(l\left(\nabla h^{\left(p_{y}\right)}(l)\right)^{2}\right)_{z}-\frac{N}{4} l\left(\left(\nabla h^{\left(p_{y}\right)}(l)\right)^{2}\right)_{z}=\frac{(-1)^{N}}{4}<D l, D\left(\nabla h^{\left(p_{y}\right)}(l)\right)^{2}>,
$$

where $\tilde{l}:=l d z \in \tilde{\mathscr{G}}_{(1 \mid N)}^{*}, \nabla h^{(y)}(\tilde{l}):=\nabla h^{(y)}(l) \partial / \partial z \in \tilde{\mathscr{G}}_{(1 \mid N)}$. If the corresponding gradient has the asymptotic expansion as $|z| \rightarrow \infty$

$$
\nabla h^{\left(p_{y}\right)}(\tilde{l}) \simeq\left(V_{2} z^{2}+V_{1} z+V_{0}+V_{-1} z^{-1}+V_{-2} z^{-2}+\ldots\right) \partial / \partial z
$$

where $p_{y}=2, V_{j} \in C^{2}\left(\mathbb{R}^{2} \times \Lambda_{1}^{N} ; \Lambda_{0}\right), j \in \mathbb{Z}, j \leq 2$, are functional parameters, we can construct the Hamiltonian flow

$$
d l / d y=-l_{z} \nabla h^{(y)}(l)-\frac{4-N}{2} l\left(\nabla h^{(y)}(l)\right)_{z}+\frac{(-1)^{N}}{2}<D l, D \nabla h^{(y)}(l)>
$$

in the framework of the classical AKS-theory. The constant Casimir invariant $h^{\left(p_{t}\right)} \in \mathrm{I}\left(\tilde{\mathscr{G}}_{(1 \mid N)}^{*}\right), p_{t}=0$, generates the trivial flow

$$
d l / d t=0
$$

The compatibility condition of these two flows for all $y, t \in \mathbb{R}$ is equivalent to the following system of two a priori compatible linear vector field equations

$$
\begin{aligned}
& \frac{\partial \psi}{\partial y}+\nabla h^{(y)}(l) \frac{\partial \psi}{\partial z}+\frac{1}{2}<D \nabla h^{(y)}(l), D \psi>=0 \\
& \frac{\partial \psi}{\partial t}+\nabla h^{(t)}(l) \frac{\partial \psi}{\partial z}+\frac{1}{2}<D \nabla h^{(t)}(l), D \psi>=0
\end{aligned}
$$

or

$$
\frac{\partial \psi}{\partial y}+V \frac{\partial \psi}{\partial z}+\frac{1}{2}<D V, D \psi>=0, \frac{\partial \psi}{\partial t}=0
$$

where $\nabla h^{(y)}(l):=V, V=V(y, t, \vartheta ; z)=V_{2} z^{2}+V_{1} z+V_{0}$, and $\nabla h^{(t)}(l)=0$, for a smooth function $\psi \in C^{2}\left(\mathbb{R}^{2} \times \Lambda_{1}^{N} ; \Lambda_{0}\right)$. In this case we have the evolutions

$$
\begin{gathered}
\frac{d z}{d y}=V-\frac{1}{2}<\theta, D V>, \quad \frac{d \vartheta}{d y}=\frac{1}{2}(D V), \\
\frac{d z}{d t}=0, \quad \frac{d \theta}{d t}=0 .
\end{gathered}
$$

Under the diffeomorphism $z \mapsto z-\alpha-<\theta, \eta>:=\lambda$ and $\vartheta \mapsto \vartheta+\eta:=\tilde{\vartheta}, \eta:=\left(\eta_{1}, \ldots, \eta_{N}\right)^{\top}, \tilde{\vartheta}:=\left(\tilde{\vartheta}_{1}, \ldots, \tilde{\vartheta}_{N}\right)^{\top}$, on $\mathbb{T}_{\mathbb{C}}^{1 \mid N}$, generated by the functions $\alpha:=\alpha(y, t) \in C^{3}\left(\mathbb{R}^{2} ; \Lambda_{0}\right)$ and $\eta:=\eta(y, t) \in C^{3}\left(\mathbb{R}^{2} ; \Lambda_{1}^{N}\right)$, the equations (4.38) are rewritten as

$$
\begin{aligned}
& \frac{\partial \psi}{\partial y}+W \frac{\partial \psi}{\partial \lambda}+\frac{1}{2}<\tilde{D} W, \tilde{D} \psi>=0 \\
& \frac{\partial \psi}{\partial t}-U \frac{\partial \psi}{\partial \lambda}-\frac{1}{2}<\tilde{D} U, \tilde{D} \psi>=0
\end{aligned}
$$

where $W:=W(y, t, \tilde{\vartheta} ; \lambda)=W_{2} \lambda^{2}+W_{1} \lambda+W_{0}, U:=U(y, t, \tilde{\vartheta}), \tilde{D}:=\left(D_{\vartheta_{1}}, \ldots, D_{\vartheta_{N}}\right)^{\top}$ and $D_{\tilde{\vartheta}_{i}}:=\frac{\partial}{\partial \tilde{\vartheta}_{i}}+\tilde{\vartheta}_{i} \frac{\partial}{\partial \lambda}, i=\overline{1, N}$. Taking into account the equations (4.39) and

$$
\begin{array}{cl}
\frac{d \lambda}{d y}=W-\frac{1}{2}<\tilde{\theta}, \tilde{D} W>, & \frac{d \tilde{\vartheta}}{d y}=\frac{1}{2}(\tilde{D} W), \\
\frac{d \lambda}{d t}=-U+\frac{1}{2}<\tilde{\theta}, \tilde{D} U>, & \frac{d \tilde{\vartheta}}{d t}=-\frac{1}{2}(\tilde{D} U),
\end{array}
$$

one obtains the function $W$ in the following form

$$
\begin{array}{r}
W=\tilde{V}+<\eta, \tilde{D} \tilde{V}>-\frac{\partial \alpha}{\partial y}+<\eta, \frac{\partial \eta}{\partial y}>, \\
\tilde{V}:=\tilde{V}(y, t, \tilde{\vartheta} ; \lambda)=\left.V(y, t, \vartheta ; z)\right|_{z=\lambda+\alpha+<\theta, \eta>, \vartheta=\tilde{\vartheta}-\eta} .
\end{array}
$$

Here the superderivatives transform by the rules

$$
D_{\vartheta_{i}}=D_{\tilde{\vartheta}_{i}}-2 \eta_{i} \partial / \partial \lambda, \quad i=\overline{1, N}
$$


and the functions $\alpha$ and $\eta$ obey the relationships

$$
\frac{\partial \alpha}{\partial t}-<\eta, \frac{\partial \eta}{\partial t}>=U, \quad \frac{\partial \eta}{\partial t}=-\frac{1}{2}(\tilde{D} U)
$$

If $W_{2}:=1$ and $U:=1 / 2 \exp \varphi, \varphi:=\varphi(y, t, \vartheta)$, the compatibility condition for the first order partial differential equations (4.40) leads to the following Lax-Sato integrable superanalogs of the Liouville heavenly type equations:

$$
\varphi_{y t}=\exp \varphi-\frac{1}{4} \sum_{i=1}^{N}\left(\frac{\partial}{\partial \tilde{\vartheta}_{i}} \varphi_{y}\right)\left(\frac{\partial}{\partial \tilde{\vartheta}_{i}} \exp \varphi\right)
$$

for $W_{0}=1$, and

$$
\begin{array}{r}
\varphi_{y t}-\varphi_{t t}=\exp \varphi- \\
-\frac{1}{4} \sum_{i=1}^{N}\left(\frac{\partial}{\partial \tilde{\vartheta}_{i}}\left(\varphi_{y}-\varphi_{t}\right)\right)\left(\frac{\partial}{\partial \tilde{\vartheta}_{i}} \exp \varphi\right)
\end{array}
$$

for $W_{0}=-1 / 2 \exp \varphi$. Owing to the relationship (4.33), the element $\tilde{l} \in \tilde{\mathscr{G}}_{(1 \mid N)}^{*}$ can be found explicitly. For example, in the case of $N=1$ it has the form

$$
\begin{array}{r}
l\left(y, t, \vartheta_{1} ; z\right)=z^{-4}\left(\vartheta_{1}\left(1-2 v_{1} z^{-1}+\left(3 v_{1}^{2}-2 v_{0}\right) z^{-2}\right)+\right. \\
\left.+\beta_{1} / 2+\left(\beta_{0} / 4-9 \beta_{1} v_{1} / 8\right) z^{-1}\right),
\end{array}
$$

where $V_{2}:=1$ and $V_{j}:=v_{j}+\vartheta_{1} \beta_{j}, j=\overline{0,1}$. Whence, one has the following proposition.

Proposition 4.2. The super Liouville equations (4.44) and (4.45) are Hamiltonian flows on the co-adjoint space $\tilde{\mathscr{G}}_{(1 \mid N)}^{*}$, generated by the seed element (4.41) and is equivalently representable as the Lax-Sato compatible linear system (4.40) on the space $C^{2}\left(\mathbb{R}^{2} \times \Lambda_{1}^{N} ; \Lambda_{0}\right)$.

\section{Integrability, bi-Hamiltonian structures and the classical Lagrange-d'Alembert principle}

It is evident that all evolution flows like (2.16) or (2.20) are Hamiltonian with respect to the second Lie-Poisson bracket (2.10) on the adjoint loop space $\tilde{\mathscr{G}}^{*}=\widetilde{\operatorname{diff}}^{*}\left(\mathbb{T}_{\mathbb{C}}^{n}\right)$. Moreover, they are poly-Hamiltonian on the corresponding functional manifolds, as the related bilinear form (2.2) is marked by integers $s \in \mathbb{Z}$. This leads to [75] an infinite hierarchy of compatible Poisson structures on the phase spaces, isomorphic, respectively, to the orbits of a chosen seed element $\tilde{l} \in \tilde{\mathscr{G}}^{*}$ or of a seed element $\tilde{l} \in \tilde{\mathscr{G}}^{*}$. Since all these Hamiltonian flows possess an infinite hierarchy of commuting nontrivial conservation laws, one can prove their formal complete integrability under naturally formulated constraints. The corresponding analytical expressions for the infinite hierarchy of conservation laws can be retrieved from the asymptotic expansion (2.14) for Casimir functional gradients by employing the well-known [6, 5, 45, 75] formal homotopy technique.

In his book "Mecanique analytique", v.1-2, published in 1788 in Paris, Lagrange formulated one of the basic, most general, differential variational principles of classical mechanics, expressing necessary and sufficient conditions for the correspondence of the real motion of a system of material points, subjected to ideal constraints and applied active forces. Within the d'Alembert-Lagrange principle the positions of the system in its real motion are compared with infinitely close positions permitted by the constraints at a given time.

According to the d'Alembert-Lagrange principle, during a real motion of a system of $N \in \mathbb{Z}_{+}$particles with masses $m_{j} \in \mathbb{R}_{+}, j=\overline{1, N}$, the totality elementary work performed by the given active forces $F^{(j)}, j=\overline{1, N}$, and by the forces of inertia for all the possible particle displacements $\delta x^{(j)} \in \mathbb{E}^{3}, j=\overline{1, N}$, is equal to or less than zero:

$$
\sum_{j=\overline{1, N}}<F^{(j)}-m_{j} \frac{d^{2} x^{(j)}}{d t^{2}}, \delta x^{(j)}>\leq 0
$$

at any moment of time $t \in \mathbb{R}$, where $\langle\cdot, \cdot\rangle$ denotes the standard scalar product in the three-dimensional Euclidean space $\mathbb{E}^{3}$. Equality in (5.1) is valid for the possible reversible displacements, the symbol $\leq$ is valid for the possible irreversible displacements $\delta x^{(j)} \in \mathbb{E}^{3}, j=\overline{1, N}$. Equation (5.1) is the general equation of the dynamics of systems with ideal constraints; it comprises all the equations and laws of motion, so that one can say that all dynamics is reduced to this single general formula.

This principle was established by J.L. Lagrange by generalizing the principle of virtual displacements with the aid of the classical d'Alembert principle. For systems subject to bilateral constraints, Lagrange used the formula (5.1) to deduce the general properties and laws of motion of bodies, as well as the equations of motion, which he applied to solve a number of problems in dynamics including the problems of motions of non-compressible, compressible and elastic liquids, thus combining "dynamics and hydrodynamics as branches of the same principle and as conclusions drawn from a single general formula".

As was first demonstrated in [31], in the last case of generalized reversible motions of a compressible elastic liquid in a simply-connected open domain $\Omega_{t} \subset \mathbb{R}^{n}$ with the smooth boundary $\partial \Omega_{t}, t \in \mathbb{R}$, in space $\mathbb{R}^{n}, n \in \mathbb{Z}_{+}$, the expression (5.1) can be rewritten as

$$
\delta W(t):=\int_{\Omega_{t}}<l(x(t) ; \lambda), \delta x(t)>d^{n} x(t)=0
$$

for all $t \in \mathbb{R}$. Here $l(x(t) ; \lambda) \in \tilde{T}^{*}\left(\mathbb{R}^{n}\right) \simeq \tilde{\mathscr{G}}^{*}$ is the corresponding virtual vector "reaction force", exerted by the ambient medium on the liquid and called a seed element, which is here assumed to depend meromorphically on a constant complex parameter $\lambda \in \mathbb{C}$. If we suppose that the evolution of liquid points $x(t) \in \Omega_{t}$ is determined for any parameters $\lambda \neq \mu \in \mathbb{C}$ by the generating gradient type vector field

$$
\frac{d x(t)}{d t}=\frac{\mu}{\mu-\lambda} \nabla h(l(\mu))(t ; x(t))
$$


and the Cauchy data

$$
\left.x(t)\right|_{t=0}=x^{(0)} \in \Omega_{0}
$$

for an arbitrarily chosen open one-connected domain $\Omega_{0} \subset \mathbb{T}^{n}$ with the smooth boundary $\partial \Omega_{0} \subset \mathbb{R}^{n}$ and a smooth functional $h: \tilde{T}^{*}\left(\mathbb{R}^{n}\right) \rightarrow \mathbb{R}$, the Lagrange-d'Alembert principle says: the infinitesimal virtual work (5.2) equals zero for all moments of time, that is $\delta W(t)=0=$ $\delta W(0)$ for all $t \in \mathbb{R}$. To check this, let us calculate the temporal derivative of the expression (5.2):

$$
\begin{gathered}
\frac{d}{d t} \delta W(t)=\frac{d}{d t} \int_{\Omega_{t}}<l(x(t) ; \lambda), \delta x(t)>d^{n} x(t)= \\
=\frac{d}{d t} \int_{\Omega_{0}}<l(x(t) ; \lambda), \delta x(t)>\left|\frac{\partial(x(t)}{\partial x_{0}}\right| d^{n} x^{(0)}=\int_{\Omega_{0}} \frac{d}{d t}\left(<l(x(t) ; \lambda), \delta x(t)>\left|\frac{\partial(x(t)}{\partial x_{0}}\right|\right) d^{n} x^{(0)}= \\
=\int_{\Omega_{0}}\left[\frac{d}{d t}<l(x(t) ; \lambda), \delta x(t)>+<l(x(t) ; \lambda), \delta x(t)>\operatorname{div} \tilde{K}(\mu)\right]\left|\frac{\partial(x(t)}{\partial x_{0}}\right| d^{n} x^{(0)}= \\
=\int_{\Omega_{t}}\left[\frac{d}{d t}<l(x(t) ; \lambda), \delta x(t)>+<l(x(t) ; \lambda), \delta x(t)>\operatorname{div} \tilde{K}(\mu)\right] d^{n} x(t)=0,
\end{gathered}
$$

if the condition

$$
\frac{d}{d t}<l(x(t) ; \lambda), \delta x(t)>+<l(x(t) ; \lambda), \delta x(t)>\operatorname{div} \tilde{K}(\mu ; \lambda)=0
$$

holds for all $t \in \mathbb{R}$, where

$$
\tilde{K}(\mu ; \lambda):=\frac{\mu}{\mu-\lambda} \nabla h(\tilde{l}(\mu))=\frac{\mu}{\mu-\lambda}<\nabla h(l(\mu)), \frac{d}{d x}>
$$

is a vector field on $\mathbb{R}^{n}$, corresponding to the evolution equations (5.3). Taking into account that the full temporal derivative $d / d t:=$ $\partial / \partial t+L_{\tilde{K}(\mu ; \lambda)}$, where $L_{\tilde{K}(\mu ; \lambda)}=i_{\tilde{K}(\mu ; \lambda)} d+d i_{\tilde{K}(\mu ; \lambda)}$ denotes the well known [1, 5, 29] Cartan expression for the Lie derivation along the vector field (5.6), can be represented as $\mu, \lambda \rightarrow \infty,|\lambda / \mu|<1$ in the asymptotic form

$$
\frac{d}{d t} \sim \sum_{j \in \mathbb{Z}_{+}} \mu^{-j} \frac{\partial}{\partial t_{j}}+\sum_{j \in \mathbb{Z}_{+}} \mu^{-j} L_{\tilde{K}_{j}(\lambda,},
$$

the equality (5.5) can be equivalently rewritten as an infinite hierarchy of the following evolution equations

$$
\partial \tilde{l}(\lambda) / \partial t_{j}:=-a d_{\tilde{K}_{j}(\lambda)_{+}}^{*} \tilde{l}(\lambda)
$$

for every $j \in \mathbb{Z}_{+}$on the space of differential 1 -forms $\tilde{\Lambda}^{1}\left(\mathbb{R}^{n}\right) \simeq \widetilde{\mathscr{G}} \tilde{\mathscr{G}}^{*}$, where $\tilde{l}(\lambda):=<l(x ; \lambda), d x>\in \tilde{\Lambda}^{1}\left(\mathbb{R}^{n}\right) \simeq \tilde{\mathscr{G}} \tilde{\mathscr{G}}^{\tilde{\mathscr{G}}}:=\widetilde{\operatorname{dif} f}\left(\mathbb{R}^{n}\right)$ is the Lie algebra of the corresponding loop diffeomorphism group $\widetilde{\operatorname{Diff}}\left(\mathbb{R}^{n}\right)$. From (5.6) one easily finds that

$$
\tilde{K}_{j}(\lambda)=\nabla h^{(j)}(\tilde{l})
$$

for $\lambda \in \mathbb{C}$ and any $j \in \mathbb{Z}_{+}$, the evolution equations (5.8) transform equivalently into

$$
\partial \tilde{l}(\lambda) / \partial t_{j}:=-a d_{\nabla h^{(j)}}^{*}(\tilde{l})_{+} \tilde{l}(\lambda)
$$

allowing to formulate the following important Adler-Kostant-Symes type [6, 5, 7, 75, 62, 61] proposition.

Proposition 5.1. The evolution equations (5.10) are completely integrable mutually commuting Hamiltonian flows on the adjoint loop space $\tilde{\mathscr{G}}^{*}$ for a seed element $\tilde{l}(\lambda) \in \tilde{\mathscr{G}}^{*}$, generated by Casimir functionals $h^{(j)} \in \mathrm{I}\left(\tilde{\mathscr{G}}^{*}\right)$, naturally determined by conditions ad $d^{*} h^{(j)}(\tilde{l})$ l $(\lambda)=0$, $j \in \mathbb{Z}_{+}$, with respect to the modified Lie-Poisson bracket on the adjoint space $\tilde{\mathscr{G}}^{*}$

$$
\{(\tilde{l}, \tilde{X}),(\tilde{l}, \tilde{Y})\}:=\left(\tilde{l},[\tilde{X}, \tilde{Y}]_{\mathscr{R}}\right),
$$

defined for any $\tilde{X}, \tilde{Y} \in \tilde{\mathscr{G}}$ by means of the canonical $\mathscr{R}$-structure on the loop Lie algebra $\tilde{\mathscr{G}}$ :

$$
[\tilde{X}, \tilde{Y}]_{\mathscr{R}}:=\left[\tilde{X}_{+}, \tilde{Y}_{+}\right]-\left[\tilde{X}_{-}, \tilde{Y}_{-}\right],
$$

where " $\tilde{Z}_{ \pm} "$ means the positive $(+) /(-)$-negative part of a loop Lie algebra element $\tilde{Z} \in \tilde{\mathscr{G}}$ subject to the loop parameter $\lambda \in \mathbb{C}$.

If, for instance, we consider the first two flows from (5.10) in the form

$$
\begin{array}{ll}
\partial \tilde{l}(\lambda) / \partial t_{1} & :=\partial \tilde{l}(\lambda) / \partial y=-a d_{\nabla h^{(y)}(\tilde{l})}^{*} \tilde{l}(\lambda), \\
\partial \tilde{l}(\lambda) / \partial t_{2} & :=\partial \tilde{l}(\lambda) / \partial t=-a d_{\nabla h^{(t)}(\tilde{l})}^{*} \tilde{l}(\lambda),
\end{array}
$$

where

$$
\nabla h^{(y)}(\tilde{l}):=\left.\nabla h^{(1)}(\tilde{l})\right|_{+}, \quad \nabla h^{(t)}(\tilde{l}):=\left.\nabla h^{(t)}(\tilde{l})\right|_{+},
$$

which are by construction commuting, from their compatibility condition one obtains a system of nonlinear partial differential equations for the coefficients of the seed element $\tilde{l}(\lambda) \in \tilde{\mathscr{G}}^{*}$. As this system is equivalent to the Lax-Sato compatibility condition for the corresponding vector fields $\nabla h^{(y)}(\tilde{l})$ and $\nabla h^{(t)}(\tilde{l}) \in \tilde{\mathscr{G}}$ :

$$
\left[\partial / \partial y+\nabla h^{(y)}(\tilde{l}), \partial / \partial t+\nabla h^{(t)}(\tilde{l})\right]=0
$$

from (5.13) we obtain a system of nonlinear equations in partial derivatives (often called heavenly) that was analyzed in a series of articles $[39,9,46,47,48,65,66,42,73,74]$ and more recently in $[11,9,10,33]$. These works are closely related to the problem of constructing a hierarchy of commuting vector fields, analytically depending on a complex parameter $\lambda \in \mathbb{C}$, which was posed in 1928 by the French mathematician M.A. Buhl [13, 14, 15] and in general form studied and completely solved by M.G. Pfeiffer in [50, 51, 52, 53, 54, 55]. 


\section{Acknowledgements}

The authors cordially thank Prof. M. Błaszak, Prof. J. Cieślinski, Prof. A. Sym and Prof. A. Samoilenko for their cooperation and useful discussions of the results in this paper during the Workshop "Nonlinearity and Geometry", 20-23 January, 2017 in Warsaw, Poland, and the International Conference in Functional Analysis dedicated to the $125^{\text {th }}$ anniversary of Stefan Banach, 18 - 23 September, 2017 in Lviv, Ukraine. A.P. is especially indebted to Prof. M. Pavlov, Prof. W.K. Schief and Prof. Ya. G. Prytula for mentioning important references, which were very helpful in preparing the manuscript. He is also greatly indebted to Prof. V.E. Zakharov (University of Arizona, Tucson) and Prof. J. Szmigelski (University of Saskatchewan, Saskatoon) for their interest in the work and instructive discussions during the XXXV Workshop on Geometric Methods in Physics, 26.06-2.07.2016 in Białowieża, Poland. A.P.'s last but not least thanks go also to the Department of Mathematical Sciences of the NJIT (Newark NJ, USA) for the invitation to visit the NJIT during the Summer of 2017, where an essential part of this paper was completed.

\section{References}

[1] R. Abraham and J. Marsden, Foundations of Mechanics, Second Edition, Benjamin Cummings, NY

[2] L.M. Alonso, A.B. Shabat, Hydrodynamic reductions and solutions of a universal hierarchy, Theor. Math. Phys,104 (2004), 1073-1085

[3] V.I. Arnold, Mathematical Methods of Classical Mechanics., Springer, NY, 1978

[4] D. Blackmore, A.K. Prykarpatsky, Dark Equations and Their Light Integrability, Journal of Nonlinear Mathematical Physics, Vol. 21, No. 3 (2014), 407-428

[5] D. Blackmore, A.K. Prykarpatsky and V.H. Samoylenko, Nonlinear dynamical systems of mathematical physics, World Scientific Publisher, NJ, USA,

[6] M. Błaszak, Classical R-matrices on Poisson algebras and related dispersionless systems, Phys. Lett. A 297(3-4) (2002) 191-195

[7] M. Błaszak and B.M. Szablikowski, Classical R-matrix theory of dispersionless systems: II. (2 + 1) dimension theory, J. Phys. A: Math. Gen. 35 (2002), 10345

[8] L.V. Bogdanov, Interpolating differential reductions of multidimensional integrable hierarchies, TMF, 2011, Volume 167, Number 3, 354-363

[9] L.V. Bogdanov, V.S. Dryuma, S.V. Manakov, Dunajski generalization of the second heavenly equation: dressing method and the hierarchy, J. Phys. A: Math. Theor. 40 (2007), 14383-14393

[10] L.V. Bogdanov, B.G. Konopelchenko, On the heavenly equation and its reductions, J. Phys. A, Math. Gen. 39 (2006), 11793-11802

[11] L.V. Bogdanov, M.V. Pavlov, Linearly degenerate hierarchies of quasiclassical SDYM type, arXiv:1603.00238v2 [nlin.SI] 15 Mar 2016

[12] A.J. Bruce, K. Grabowska, J. Grabowski, Remarks on contact and Jacobi geometry. SIGMA 13, 059 (2017);(arXiv:1507.05405)

[13] M.A. Buhl, Surles operateurs differentieles permutables ou non, Bull. des Sc.Math.,1928, S.2, t. LII, p. 353-361

[14] M.A. Buhl, Apercus modernes sur la theorie des groupes continue et finis, Mem. des Sc. Math., fasc. XXXIII, Paris, 1928

[15] M.A. Buhl, Apercus modernes sur la theorie des groupes continue et finis, Mem. des Sc. Math., fasc. XXXIII, Paris, 1928

[16] P.A. Burovskiy, E.V. Ferapontov, S.P. Tsarev, Second order quasilinear PDEs and conformal structures in projective space, Int. J. Math. 21 (2010), no. 6, 799-841, arXiv:0802.2626

[17] A. Cartan, Differential Forms. Dover Publisher, USA, 1971

[18] Earl A. Coddington, Norman Levinson, Theory of ordinary differential equations, International series in pure and applied mathematics, McGraw-Hill,

[19] B.A. Dubrovin, S.P. Novikov and A.T. Fomenko, Modern Geometry - Methods and Applications: Part I: The Geometry of Surfaces, Transformation Groups, and Fields (Graduate Texts in Mathematics) 2nd ed., Springer, Berlin, 1992

[20] M. Dunajski, Anti-self-dual four-manifolds with a parallel real spinor, Proc. Roy. Soc. A, 458 (2002), 1205

[21] M. Dunajski, E.V. Ferapontov and B. Kruglikov, On the Einstein-Weyl and conformal self-duality equations. arXiv: $1406.0018 \mathrm{v} 3$ [nlin.SI] 29 Jun 2015

[22] M. Dunajski, W. Kryński, Einstein-Weyl geometry, dispersionless Hirota equation and Veronese webs, arXiv:1301.0621

[23] M. Dunajski, L.J. Mason, P. Tod, Einstein-Weyl geometry, the dKP equation and twistor theory, J. Geom. Phys. 37 (2001), N1-2, 63-93

[24] E.V. Ferapontov and B. Kruglikov, Dispersionless integrable systems in 3D and Einstein-Weyl geometry. arXiv:1208.2728v3 [math-ph] 9 May 2013

[25] E.V. Ferapontov, B. Kruglikov, V. Novikov, Integrability of dispersionless Hirota type equations and the symplectic Monge-Amp'ere property. rXiv: $1707.08070 \mathrm{v} 2$ [nlin.SI] 9 Jan 2018

[26] E.V. Ferapontov and J. Moss, Linearly degenerate PDEs and quadratic line complexes, arXiv:1204.2777v1 [math.DG] 12 Apr 2012

[27] J. Gibbons and S.P. Tsarev, Reductions of the Benney Equations, Physics Letters A, Vol. 211, Issue 1, Pages 19-24, 1996

[28] J. Gibbons, S.P. Tsarev, Conformal maps and reductions of the Benney equations, Phys. Lett. A, 258 (1999) $263-270$.

[29] C. Godbillon, Geometrie Differentielle et Mecanique Analytique. Hermann Publ., Paris, 1969

[30] M. Gromov, Partial differential relations, Ergebnisse der Mathematik und ihrer Grenzgebiete, Vol. 9, Springer-Verlag, Berlin, Heidelberg, New York, $363 \mathrm{p}$.

[31] O.E. Hentosh, Y.A. Prykarpatsky, D. Blackmore and A.K. Prykarpatski, Lie-algebraic structure of Lax-Sato integrable heavenly equations and the Lagrange-d'Alembert principle, Journal of Geometry and Physics, 120 (2017), 208-227

[32] A. A. Kirillov, Local Lie algebras, Uspekhi Mat. Nauk, 31 (1976), N4(190), 57-76

[33] B.G. Konopelchenko, Grassmanians $\operatorname{Gr}(N-1, N+1)$, closed differential $N-1$ forms and $N$-dimensional integrable systems. arXiv: $1208.6129 v 2$ [nlin.SI] 5 Mar 2013

[34] I. Krasil'shchik, A natural geometric construction underlying a class of Lax pairs, Lobachevskii J. of Math., 37 (2016) no. 1, 61-66; arXiv:1401.0612

[35] I.S. Krasil'shchik1, A. Sergyeyev, O.I. Morozov, Infinitely many nonlocal conservation laws for the ABC equation with $A+B+C \neq 0$, arXiv:1511.09430v1 [nlin.SI] 30 Nov 2015

[36] B.A. Kupershmidt, Dark equations, J. Nonlin. Math. Phys. 8 (2001), 363-445

[37] B.A. Kupershmidt, Mathematics of dispersive water waves, Commun. Math. Phys., 99 (1985), 51-73

[38] A. Kushner, V. Lychagin, V. Rubtsov, Contact geometry and non-linear differential equations. Cambridge Univ. Press, Cambridge (2007)

[39] S.V. Manakov, P.M. Santini, On the solutions of the second heavenly and Pavlov equations, J. Phys. A: Math. Theor. 42 (2009), 404013 (11pp)

[40] O.I. Mokhov, Symplectic and Poissonian geometry on loop spaces of smooth manifolds and integrable equations. Institute for Computer Studies Publ.,

Moscow-Izhevsk, 2004 (in Ruissian)
[41] O.I. Morozov, A two-Component generalization of the integrable rd-Dym equation, SIGMA, 8 (2012), $051-056$.

[42] O.I. Morozov, A. Sergyeyev, The four-dimensional Martinez-Alonso-Shabat equation: reductions, nonlocal symmetries, and a four-dimensional integrable generalization of the ABC equation, Preprint submitted to JGP, 2014, $11 \mathrm{p}$.

[43] V. G. Mikhalev, On the Hamiltonian formalism for Korteweg-de Vries type hierarchies, Funct. Anal. Appl., 26, Issue 2 (1992), 140-142

[44] S.P. Novikov (Editor), Theory of solitons: the Inverse Scattering Method. Springer, 1984

[45] P. Olver, Applications of Lie Groups to Differential Equations, Second Edition, Springer-Verlag, New York, 1993

[46] V. Ovsienko, Bi-Hamilton nature of the equation $u_{t x}=u_{x y} u_{y}-u_{y y} u_{x}$, arXiv:0802.1818v1 [math-ph] 13 Feb 2008

[47] V. Ovsienko, C. Roger, Looped Cotangent Virasoro Algebra and Non-Linear Integrable Systems in Dimension $2+1$, Commun. Math. Phys. 273 (2007), 357-378

[48] M.V. Pavlov, Integrable hydrodynamic chains, J. Math. Phys. 44 (2003), Issue 9, 4134-4156

[49] M.V. Pavlov, Classification of integrable Egorov hydrodynamic chains, Theoret. and Math. Phys. 138 (2004), 45-58, nlin.SI/0603055

[50] G. Pfeiffer, Generalisation de la methode de Jacobi pour l'integration des systems complets des equations lineaires et homogenes, Comptes Rendues de l'Academie des Sciences de l'URSS, 1930, t. 190, p. 405-409

[51] M. G. Pfeiffer, Sur la operateurs d'un systeme complet d'equations lineaires et homogenes aux derivees partielles du premier ordre d'une fonction inconnue, Comptes Rendues de l'Academie des Sciences de l'URSS, 1930, t. 190, p. 909-911

[52] M. G. Pfeiffer, La generalization de methode de Jacobi-Mayer, Comptes Rendues de l'Academie des Sciences de 1'URSS, 1930, t. 191, p. 1107-1109

[53] M. G. Pfeiffer, Sur la permutation des solutions s'une equation lineaire aux derivees partielles du premier ordre, Bull. des Sc. Math., 1928, S.2,t.LII, p. 353-361 
[54] M. G. Pfeiffer, Quelques additions au probleme de M. Buhl, Atti dei Congresso Internationale dei Matematici, Bologna, 1928, t.III, p. 45-46

[55] M. G. Pfeiffer, La construction des operateurs d'une equation lineaire, homogene aux derivees partielles premier ordre, Journal du Cycle Mathematique, Academie des Sciences d'Ukraine, Kyiv, N1, 1931, p. 37-72 (in Ukrainian)

[56] J.F. Plebański, Some solutions of complex Einstein equations, J. Math. Phys. 16 (1975), Issue 12, 2395-2402

[57] A. Presley and G. Segal, Loop groups, Oxford Mathematical Monographs, Oxford Univresity Press, 1986.

[58] N.K. Prykarpatska, D. Blackmore, V.H. Samoylenko, E. Wachnicki, M. Pytel-Kudela, The Cartan-Monge geometric approach to the characteristic method for nonlinear partial differential equations of the first and higher orders. Nonlinear Oscillations, 10(1) (2007), 22-31

[59] A.K. Prykarpatsky I.V. Mykytyuk, Algebraic integrability of nonlinear dynamical systems on manifolds: classical and quantum aspects. Kluwer Academic Publishers, the Netherlands, 1998

[60] Ya.A. Prykarpatsky and A.K. Prykarpatski, The integrable heavenly type equations and their Lie-algebraic structure, arXiv:1785057 [nlin.SI] 24 Jan 2017

[61] Y.A. Prykarpatsky and A.M. Samoilenko, Algebraic - analytic aspects of integrable nonlinear dynamical systems and their perturbations. Kyiv, Inst. Mathematics Publisher, v. 41, 2002 (in Ukrainian)

[62] A.G. Reyman, M.A. Semenov-Tian-Shansky, Integrable Systems, The Computer Research Institute Publ., Moscow-Izhvek, 2003 (in Russian)

[63] M. Sato, Y. Sato, Soliton equations as dynamical systems on infinite dimensional Grassmann manifold, in Nonlinear Partial Differential Equations in Applied Science; Proceedings of the U.S.-Japan Seminar, Tokyo, 1982, Lect. Notes in Num. Anal. 5 (1982), $259-271$.

[64] M. Sato, and M. Noumi, Soliton equations and the universal Grassmann manifolds, Sophia University Kokyuroku in Math. 18 (1984) (in Japanese).

[65] W.K. Schief, Self-dual Einstein spaces via a permutability theorem for the Tzitzeica equation, Phys. Lett. A, Volume 223, Issues 1-2, 25 November 1996, 55-62

[66] W.K. Schief, Self-dual Einstein spaces and a discrete Tzitzeica equation. A permutability theorem link, In Symmetries and Integrability of Difference Equations, P. Clarkson and F. Nijhoff, eds, London Mathematical Society, Lecture Note Series 255, Cambridge University Press (1999) $137-148$.

[67] A. Sergyeyev and B. M. Szablikowski, Central extensions of cotangent universal hierarchy: (2+1)-dimensional bi-Hamiltonian systems, Phys. Lett. A, 372 (2008) 7016-7023

[68] B. Szablikowski, Hierarchies of Manakov-Santini Type by Means of Rota-Baxter and Other Identities, SIGMA 12 (2016), 022, 14 pages

[69] B. Szablikowski, M. Błaszak, Meromorphic Lax representations of (1+1)-dimensional multi-Hamiltonian dispersionless systems. J. Math. Phys.,47, N9, 2006, 092701

[70] A. Sergyeyev, A simple construction of recursion operators for multidimensional dispersionless integrable systems, J. Math. Anal. Appl. 454 (2017), no.2, 468-480; arXiv: 1501.01955

[71] A. Sergyeyev, New integrable (3+1)-dimensional systems and contact geometry, arXiv:1401.2122v4 [math.AP] 26 Sep 2017

[72] M.B. Sheftel1, A.A. Malykh, D. Yazıc1, Recursion operators and bi-Hamiltonian structure of the general heavenly equation, arXiv:1510.03666v3 [math-ph] 25 Jun 2016

[73] K. Takasaki and T. Takebe, SDiff(2) Toda equation - Hierarchy, Tau function, and symmetries, Letters in Mathematical Physics 23(3), 205-214 (1991)

[74] K. Takasaki and T. Takebe, Integrable Hierarchies and Dispersionless Limit, Reviews in Mathematical Physics 7(05), 743-808 (1995)

[75] L.A. Takhtajan, L.D. Faddeev, Hamiltonian Approach in Soliton Theory, Springer, Berlin-Heidelberg, 1987

[76] I. Zakharevich, Nonlinear wave equation, nonlinear Riemann problem, and the twistor transform of Veronese webs, arXiv:math-ph/0006001 